\title{
Studies of Substituent Effect on Asymmetric Epoxidation of Chromenes by Chiral Dioxirane
}

\author{
O. Andrea Wong and Yian Shi* \\ Department of Chemistry \\ Colorado State University \\ Fort Collins, CO 80523 \\ Phone: 970-491-7424 \\ Fax: 970-491-1801 \\ Email: yian@lamar.colostate.edu
}

\section{Supporting Information}

\section{Table of Contents}

General Methods

Characterization data of ketone $\mathbf{5}$

Characterization data of epoxides

S-2

HPLC and GC data for the determination of the ee's

NMR spectral data of ketone $\mathbf{5}$

NMR spectral data of selected epoxides (Table 1, entries 3, 4, 10, 11, and 17) 
General Methods. All commercially available reagents were used without further purification. All glassware used for the epoxidation was carefully washed with soap water to be free of any trace metals which catalyze the decomposition of Oxone. Column chromatography was performed with silica gel (200-400 mesh). Solvents were evaporated under reduced pressure.

${ }^{1} \mathrm{H}$ NMR spectra were recorded on $400 \mathrm{MHz}$ NMR spectrometer and ${ }^{13} \mathrm{C}$ NMR spectra were recorded on $100 \mathrm{MHz} \mathrm{NMR}$ spectrometer using $\mathrm{CDCl}_{3}$ as a solvent. IR spectra were recorded on a FT-IR spectrometer. Melting points were uncorrected.

\section{Characterization data of ketones 5}

White solid; mp 67-69 ${ }^{\circ} \mathrm{C} ;[\alpha]^{20}{ }_{\mathrm{D}}=-42.6\left(\mathrm{c}, 0.43, \mathrm{CHCl}_{3}\right.$ ); IR (film): 1778, $985 \mathrm{~cm}^{-1} ;{ }^{1} \mathrm{H}$ NMR (400 MHz, $\left.\mathrm{CDCl}_{3}\right) \delta 4.80(\mathrm{~d}, J=5.2 \mathrm{~Hz}, 1 \mathrm{H}), 4.60-4.54(\mathrm{~m}, 2 \mathrm{H}), 4.25-4.18(\mathrm{~m}, 2 \mathrm{H}), 3.34-3.22$ (m, 3H), 1.56-1.51 (m, 2H), $1.44(\mathrm{~s}, 3 \mathrm{H}), 1.40(\mathrm{~s}, 3 \mathrm{H}), 1.28(\mathrm{~m}, 6 \mathrm{H}), 0.87$ (t, $J=6.8 \mathrm{~Hz}, 3 \mathrm{H})$ ${ }^{13} \mathrm{C} \mathrm{NMR}\left(100 \mathrm{MHz}, \mathrm{CDCl}_{3}\right) \delta 195.5,154.2,111.1,99.9,77.6,75.6,60.9,48.9,44.2,31.5,27.3$, 26.3, 26.1, 22.6, 14.1; HRMS calcd. For $\mathrm{C}_{16} \mathrm{H}_{26} \mathrm{NO}_{6}(\mathrm{M}+1)$ 328.1760, found 328.1760.

\section{Characterization data of epoxides}

Table 1, entry 1

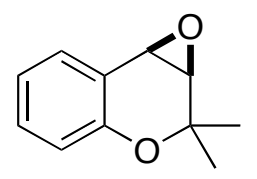

Yellow solid; mp 27-29 ${ }^{\circ} \mathrm{C} ;[\alpha]^{20}{ }_{\mathrm{D}}=+28.0\left(c, 0.82\right.$, THF) $\left(84 \%\right.$ ee); IR (film): $1491 \mathrm{~cm}^{-1} ;{ }^{1} \mathrm{H}$ NMR (400 MHz, $\left.\mathrm{CDCl}_{3}\right) \delta 7.35(\mathrm{dd}, J=7.2,1.6 \mathrm{~Hz}, 1 \mathrm{H}), 7.25$ (td, $\left.J=7.6,1.6 \mathrm{~Hz}, 1 \mathrm{H}\right), 6.94$ (td, $J=7.2,0.8 \mathrm{~Hz}, 1 \mathrm{H}), 6.82(\mathrm{~d}, J=8.0 \mathrm{~Hz}, 1 \mathrm{H}), 3.92(\mathrm{~d}, J=4.2 \mathrm{~Hz}, 1 \mathrm{H}), 3.51,(\mathrm{~d}, J=4.2 \mathrm{~Hz}, 1 \mathrm{H})$, $1.59(\mathrm{~s}, 3 \mathrm{H}), 1.27(\mathrm{~s}, 3 \mathrm{H}) ;{ }^{13} \mathrm{C} \mathrm{NMR}\left(100 \mathrm{MHz}, \mathrm{CDCl}_{3}\right) \delta 152.8,130.5,129.8,121.3,120.1$, 
118.2, 73.2, 63.1, 51.2, 25.9, 22.8; Anal. Calcd for $\mathrm{C}_{11} \mathrm{H}_{12} \mathrm{O}_{2}$ : C, 74.98; H, 6.86. Found: C, 75.20; H, 6.92 .

(a) Sasaki, H.; Irie, R.; Hamada, T.; Suzuki, K.; Katsuki, T. Tetrahedron 1994, 50, 11827.

(b) Scheurer, A.; Mosset, P.; Spiegel, M.; Saalfrank, R.W. Tetrahedron 1999, 55, 1063.

Table 1, entry 2

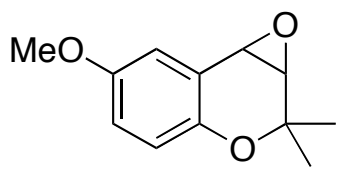

White solid; $\operatorname{mp} 61-64{ }^{\circ} \mathrm{C} ;[\alpha]^{20}{ }_{\mathrm{D}}=+12.4\left(c, 0.66, \mathrm{CHCl}_{3}\right)\left(90 \%\right.$ ee); IR (film): $1159 \mathrm{~cm}^{-1} ;{ }^{1} \mathrm{H}$ NMR $\left(400 \mathrm{MHz}, \mathrm{CDCl}_{3}\right) \delta 6.90(\mathrm{~d}, J=3.2 \mathrm{~Hz}, 1 \mathrm{H}), 6.80(\mathrm{dd}, J=8.8,2.8 \mathrm{~Hz}, 1 \mathrm{H}), 6.75(\mathrm{dd}, J=$

$8.8 \mathrm{~Hz}, 1 \mathrm{H}), 3.87(\mathrm{~d}, J=4.2 \mathrm{~Hz}, 1 \mathrm{H}), 3.79(\mathrm{~s}, 3 \mathrm{H}), 3.48(\mathrm{~d}, J=4.2 \mathrm{~Hz}, 1 \mathrm{H}), 1.57,(\mathrm{~s}, 3 \mathrm{H}), 1.23$ $(\mathrm{s}, 3 \mathrm{H}) ;{ }^{13} \mathrm{C} \mathrm{NMR}\left(100 \mathrm{MHz}, \mathrm{CDCl}_{3}\right) \delta$ 154.0, 146.4, 120.8 118.9, 115.8, 114.9, 72.9, 63.0, 56.0, 51.3, 25.9, 22.5. Anal. Calcd for $\mathrm{C}_{12} \mathrm{H}_{14} \mathrm{O}_{3}$ : C, 69.88; H, 6.84. Found: C, 70.02; H, 6.89.

(a) Scheurer, A.; Mosset, P.; Spiegel, M.; Saalfrank, R.W. Tetrahedron 1999, 55, 1063.

(b) Wang, Q.; She, X.; Ren, X.; Ma, J.; Pan, X. Tetrahedron: Asymmetry 2004, 15, 29.

\section{Table 1, entry 3}

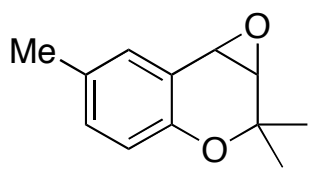

White solid; $\operatorname{mp~} 42-44{ }^{\circ} \mathrm{C} ;[\alpha]^{20}{ }_{\mathrm{D}}=+15.5\left(c, 0.73, \mathrm{CHCl}_{3}\right)\left(92 \%\right.$ ee); IR (film): $1501 \mathrm{~cm}^{-1} ;{ }^{1} \mathrm{H}$ NMR $\left(400 \mathrm{MHz}, \mathrm{CDCl}_{3}\right) \delta 7.15(\mathrm{~d}, J=2.0 \mathrm{~Hz}, 1 \mathrm{H}), 7.04(\mathrm{dd}, J=8.0,1.2 \mathrm{~Hz}, 1 \mathrm{H}), 6.71(\mathrm{~d}, J=$ $8.0 \mathrm{~Hz}, 1 \mathrm{H}), 3.87(\mathrm{~d}, J=4.4 \mathrm{~Hz}, 1 \mathrm{H}), 3.48(\mathrm{~d}, J=4.4 \mathrm{~Hz}, 1 \mathrm{H}), 2.30(\mathrm{~s}, 3 \mathrm{H}), 1.58(\mathrm{~s}, 3 \mathrm{H}), 1.24$ (s, $3 \mathrm{H}) ;{ }^{13} \mathrm{C} \mathrm{NMR}\left(100 \mathrm{MHz}, \mathrm{CDCl}_{3}\right) \delta 150.4,131.0,130.6,130.2,119.8,118.0,73.0,63.1,51.3$, 25.9, 22.7, 20.7; HRMS calcd for $\mathrm{C}_{12} \mathrm{H}_{15} \mathrm{O}_{2}(\mathrm{M}+1)$ 191.1072, found 191.1072. 
Table 1, entry 4

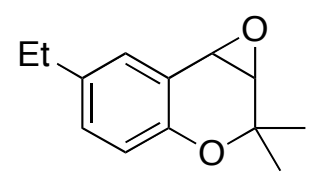

Yellow oil; $[\alpha]^{20}{ }_{\mathrm{D}}=+11.5\left(c, 0.89, \mathrm{CHCl}_{3}\right)\left(90 \%\right.$ ee); $\mathrm{IR}$ (film): $1499 \mathrm{~cm}^{-1} ;{ }^{1} \mathrm{H}$ NMR $(400$ $\left.\mathrm{MHz}, \mathrm{CDCl}_{3}\right) \delta 7.17(\mathrm{~d}, J=2.0 \mathrm{~Hz}, 1 \mathrm{H}), 7.07(\mathrm{dd}, J=8.4,2.4 \mathrm{~Hz}, 1 \mathrm{H}), 6.74(\mathrm{~d}, J=8.0 \mathrm{~Hz}, 1 \mathrm{H})$, $3.89(\mathrm{~d}, J=4.4 \mathrm{~Hz}, 1 \mathrm{H}), 3.49(\mathrm{~d}, J=4.4 \mathrm{~Hz}, 1 \mathrm{H}), 2.60(\mathrm{q}, J=7.6 \mathrm{~Hz}, 2 \mathrm{H}), 1.58(\mathrm{~s}, 3 \mathrm{H}), 1.25,(\mathrm{~s}$, $3 \mathrm{H}), 1.22(\mathrm{t}, J=7.6 \mathrm{~Hz}, 3 \mathrm{H}) ;{ }^{13} \mathrm{C} \mathrm{NMR}\left(100 \mathrm{MHz}, \mathrm{CDCl}_{3}\right) \delta 150.6,137.1,129.8,129.0,119.8$, 117.9, 73.0, 63.0, 51.3, 28.2, 25.9, 22.7, 16.0; HRMS calcd for $\mathrm{C}_{13} \mathrm{H}_{17} \mathrm{O}_{2}(\mathrm{M}+1)$ 205.1229, found 205.1225 .

\section{Table 1, entry 5}

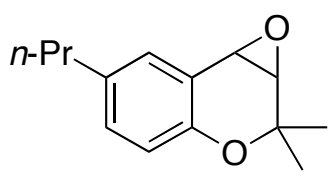

Yellow oil; $[\alpha]^{20}{ }_{D}=+11.3\left(c, 0.69, \mathrm{CHCl}_{3}\right)(91 \%$ ee $) ;$ IR (film): $1499,1167 \mathrm{~cm}^{-1} ;{ }^{1} \mathrm{H}$ NMR $\left(400 \mathrm{MHz}, \mathrm{CDCl}_{3}\right) \delta 7.14(\mathrm{~d}, J=2.0 \mathrm{~Hz}, 1 \mathrm{H}), 7.04(\mathrm{dd}, J=8.0,2.0 \mathrm{~Hz}, 1 \mathrm{H}), 6.73(\mathrm{~d}, J=8.0 \mathrm{~Hz}$, $1 \mathrm{H}), 3.88(\mathrm{~d}, J=4.4 \mathrm{~Hz}, 1 \mathrm{H}), 3.49(\mathrm{~d}, J=4.4 \mathrm{~Hz}, 1 \mathrm{H}), 2.53(\mathrm{t}, J=7.6 \mathrm{~Hz}, 2 \mathrm{H}), 1.62$ (sextet, $J=$ $7.6 \mathrm{~Hz}, 2 \mathrm{H}), 1.58,(\mathrm{~s}, 3 \mathrm{H}), 1.25(\mathrm{~s}, 3 \mathrm{H}), 0.93(\mathrm{t}, J=7.6 \mathrm{~Hz}, 3 \mathrm{H}) ;{ }^{13} \mathrm{C} \mathrm{NMR}\left(100 \mathrm{MHz}, \mathrm{CDCl}_{3}\right) \delta$ $150.6,135.5,130.4,129.6,119.7,117.9,73.0,63.0,51.4,37.3,25.9,24.9,22.7,13.9 ; \quad$ Anal. Calcd for $\mathrm{C}_{14} \mathrm{H}_{18} \mathrm{O}_{2}$ : C, 77.03; H, 8.31. Found: C, 76.94; H, 8.22.

\section{Table 1, entry 6}




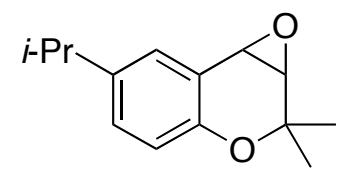

White solid; $\mathrm{mp} 27-29^{\circ} \mathrm{C} ;[\alpha]^{20}{ }_{\mathrm{D}}=+9.8\left(c, 0.99, \mathrm{CHCl}_{3}\right)(90 \%$ ee $)$; IR (film): $1498,1161 \mathrm{~cm}^{-1}$; ${ }^{1} \mathrm{H}$ NMR $\left(400 \mathrm{MHz}, \mathrm{CDCl}_{3}\right) \delta 7.19(\mathrm{~d}, J=2.0 \mathrm{~Hz}, 1 \mathrm{H}), 7.10(\mathrm{dd}, J=8.4,2.4 \mathrm{~Hz}, 1 \mathrm{H}), 6.74(\mathrm{~d}, J$ $=8.4 \mathrm{~Hz}, 1 \mathrm{H}), 3.90(\mathrm{~d}, J=4.2 \mathrm{~Hz}, 1 \mathrm{H}), 3.49(\mathrm{~d}, J=4.2 \mathrm{~Hz}, 1 \mathrm{H}), 2.86$ (septet, $J=7.2 \mathrm{~Hz}, 1 \mathrm{H}$ ), $1.58(\mathrm{~s}, 3 \mathrm{H}), 1.26,(\mathrm{~s}, 3 \mathrm{H}), 1.23(\mathrm{~d}, J=7.2 \mathrm{~Hz}, 6 \mathrm{H}) ;{ }^{13} \mathrm{C} \mathrm{NMR}\left(100 \mathrm{MHz}, \mathrm{CDCl}_{3}\right) \delta 150.6,141.8$ 128.5, 127.6, 119.6, 117.9, 73.0, 63.1, 51.5, 33.5, 25.9, 24.4, 24.3, 22.8; Anal. Calcd for $\mathrm{C}_{14} \mathrm{H}_{18} \mathrm{O}_{2}:$ C, 77.03; H, 8.31. Found: C, 76.88; H, 8.47.

Burrell, G.; Cassidy, F.; Evans, J.M.; Lightowler, D.; Stemp, G. J. Med. Chem. 1990, 33, 3023.

\section{Table 1, entry 7}

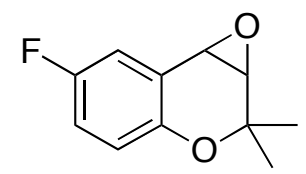

White solid; mp 38-39 ${ }^{\circ} \mathrm{C} ;[\alpha]^{20}{ }_{\mathrm{D}}=-2.04\left(c, 0.97, \mathrm{CHCl}_{3}\right)\left(89 \%\right.$ ee); IR (film): $1494 \mathrm{~cm}^{-1} ;{ }^{1} \mathrm{H}$ NMR (400 MHz, $\left.\mathrm{CDCl}_{3}\right) \delta 7.07(\mathrm{dd}, J=8.4,3.2 \mathrm{~Hz}, 1 \mathrm{H}), 6.94(\mathrm{td}, J=8.8,3.2 \mathrm{~Hz}, 1 \mathrm{H}), 6.77$ $(\mathrm{dd}, J=8.8,4.4 \mathrm{~Hz}, 1 \mathrm{H}), 3.86(\mathrm{~d}, J=4.2 \mathrm{~Hz}, 1 \mathrm{H}), 3.49(\mathrm{~d}, J=4.2 \mathrm{~Hz}, 1 \mathrm{H}), 1.58,(\mathrm{~s}, 3 \mathrm{H}), 1.24(\mathrm{~s}$, $3 \mathrm{H}) ;{ }^{13} \mathrm{C} \mathrm{NMR}\left(100 \mathrm{MHz}, \mathrm{CDCl}_{3}\right) \delta 158.4,156.0,148.7,121.4,119.33,119.36,117.1,116.9$, 116.2, 116.0, 73.3, 62.8, 50.8, 25.8, 22.5. Anal. Calcd for $\mathrm{C}_{11} \mathrm{H}_{11} \mathrm{FO}_{2}:$ C, 68.03; H, 5.71; Found: C, 67.69; H, 5.65.

\section{Table 1, entry 8}




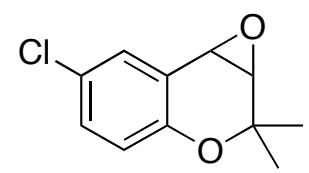

Yellow solid; mp 57-59 ${ }^{\circ} \mathrm{C} ;[\alpha]^{20}{ }_{\mathrm{D}}=+36.6\left(\mathrm{c}, 0.95, \mathrm{CHCl}_{3}\right)\left(93 \%\right.$ ee); IR (film): $1481 \mathrm{~cm}^{-1} ;{ }^{1} \mathrm{H}$ NMR $\left(400 \mathrm{MHz}, \mathrm{CDCl}_{3}\right) \delta 7.33(\mathrm{~d}, J=2.4 \mathrm{~Hz}, 1 \mathrm{H}), 7.20(\mathrm{dd}, J=8.8,2.8 \mathrm{~Hz}, 1 \mathrm{H}), 6.76(\mathrm{~d}, J=$

$8.4 \mathrm{~Hz}, 1 \mathrm{H}), 3.86(\mathrm{~d}, J=4.2 \mathrm{~Hz}, 1 \mathrm{H}), 3.50(\mathrm{~d}, J=4.2 \mathrm{~Hz}, 1 \mathrm{H}), 1.58,(\mathrm{~s}, 3 \mathrm{H}), 1.26(\mathrm{~s}, 3 \mathrm{H}) ;{ }^{13} \mathrm{C}$ NMR $\left(100 \mathrm{MHz}, \mathrm{CDCl}_{3}\right) \delta 151.4,130.4,129.4,125.9,121.8,119.6,73.6,62.8,50.6,25.8,22.7$. Anal. Calcd for $\mathrm{C}_{11} \mathrm{H}_{11} \mathrm{ClO}_{2}$ : C, 62.72; H, 5.26. Found: C, 63.00; H, 5.30.

Bulman Page, P.C; Buckley, B.R.; Heaney, H.; Blacker, A.J. Org. Lett. 2005, 7, 375.

\section{Table 1, entry 9}

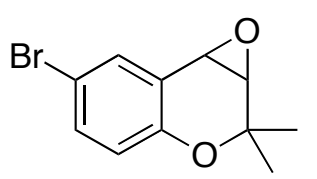

Yellow solid; mp 53-55 ${ }^{\circ} \mathrm{C} ;[\alpha]^{20}{ }_{\mathrm{D}}=+36.7\left(c, 1.06, \mathrm{CHCl}_{3}\right)\left(91 \%\right.$ ee); IR (film): $1202 \mathrm{~cm}^{-1} ;{ }^{1} \mathrm{H}$ NMR $\left(400 \mathrm{MHz}, \mathrm{CDCl}_{3}\right) \delta 7.46(\mathrm{~d}, J=2.8 \mathrm{~Hz}, 1 \mathrm{H}), 7.33(\mathrm{dd}, J=8.8,2.8 \mathrm{~Hz}, 1 \mathrm{H}), 6.70(\mathrm{~d}, J=$ $8.4 \mathrm{~Hz}, 1 \mathrm{H}), 3.85(\mathrm{~d}, J=4.4 \mathrm{~Hz}, 1 \mathrm{H}), 3.50(\mathrm{~d}, J=4.4 \mathrm{~Hz}, 1 \mathrm{H}), 1.58,(\mathrm{~s}, 3 \mathrm{H}), 1.25(\mathrm{~s}, 3 \mathrm{H}) ;{ }^{13} \mathrm{C}$ NMR $\left(100 \mathrm{MHz}, \mathrm{CDCl}_{3}\right) \delta 151.9,133.3,132.3,122.3,120.0,113.0,73.6,62.8,50.5,25.8,22.7$; Anal. Calcd for $\mathrm{C}_{11} \mathrm{H}_{11} \mathrm{BrO}_{2}$ : C, 51.79; H, 4.35. Found: C, 52.13; H, 4.39.

\section{Table 1, entry 10}<smiles>CC1(C)Oc2ccc(C#N)cc2C2OC21</smiles> 
White solid; $\mathrm{mp} 100-101{ }^{\circ} \mathrm{C} ;[\alpha]^{20}{ }_{\mathrm{D}}=+68.3\left(c, 1.05, \mathrm{CHCl}_{3}\right)(93 \%$ ee); IR (film): 2227,1491 $\mathrm{cm}^{-1} ;{ }^{1} \mathrm{H}$ NMR $\left(400 \mathrm{MHz}, \mathrm{CDCl}_{3}\right) \delta 7.66(\mathrm{~d}, J=1.6 \mathrm{~Hz}, 1 \mathrm{H}), 7.54(\mathrm{dd}, J=8.4,2.0 \mathrm{~Hz}, 1 \mathrm{H}), 6.87$ (d, $J=8.8 \mathrm{~Hz}, 1 \mathrm{H}), 3.92(\mathrm{~d}, J=4.4 \mathrm{~Hz}, 1 \mathrm{H}), 3.55(\mathrm{~d}, J=4.4 \mathrm{~Hz}, 1 \mathrm{H}), 1.61,(\mathrm{~s}, 3 \mathrm{H}), 1.31(\mathrm{~s}, 3 \mathrm{H})$;

${ }^{13} \mathrm{C}$ NMR (100 MHz, $\left.\mathrm{CDCl}_{3}\right) \delta$ 156.7, 134.7, 134.1, 121.3 119.3, 119.0, 104.6, 74.9, 62.6, 50.1, 25.8, 23.3.

(a) Scheurer, A.; Mosset, P.; Spiegel, M.; Saalfrank, R.W. Tetrahedron 1999, 55, 1063.

(b) Bulman Page, P.C; Buckley, B.R.; Heaney, H.; Blacker, A.J. Org. Lett. 2005, 7, 375.

\section{Table 1, entry 11}<smiles>Cc1ccc2c(c1)C1OC1CO2</smiles>

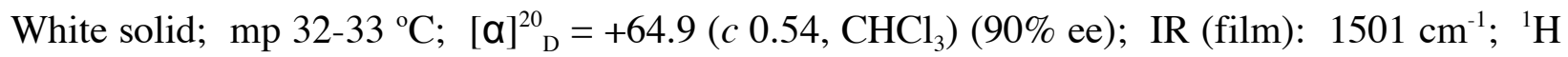
NMR $\left(400 \mathrm{MHz}, \mathrm{CDCl}_{3}\right) \delta 7.17(\mathrm{~d}, J=2.0 \mathrm{~Hz}, 1 \mathrm{H}), 7.06(\mathrm{dd}, J=8.2,1.6 \mathrm{~Hz}, 1 \mathrm{H}), 6.76(\mathrm{~d}, J=$ $8.2 \mathrm{~Hz}, 1 \mathrm{H}), 4.54(\mathrm{dd}, J=12.8,1.0 \mathrm{~Hz}, 1 \mathrm{H}), 4.11(\mathrm{~d}, J=12.8 \mathrm{~Hz}, 1 \mathrm{H}), 3.88(\mathrm{~d}, J=4.4 \mathrm{~Hz}, 1 \mathrm{H})$, $3.80(\mathrm{dd}, J=4.4,1.0 \mathrm{~Hz}, 1 \mathrm{H}), 2.31(\mathrm{~s}, 3 \mathrm{H}) ;{ }^{13} \mathrm{C} \mathrm{NMR}\left(100 \mathrm{MHz}, \mathrm{CDCl}_{3}\right) \delta 151.4,131.0,130.9$, 130.5, 120.3, 117.3, 62.9, 56.5, 49.6, 20.7. HRMS calcd for $\mathrm{C}_{10} \mathrm{H}_{11} \mathrm{O}_{2}(\mathrm{M}+1)$ 163.0759, found 163.0761 .

\section{Table 1, entry 12}

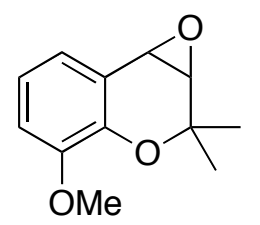

Yellow solid; $[\alpha]^{20}{ }_{D}=+23.5\left(c, 0.51, \mathrm{CHCl}_{3}\right)(82 \%$ ee $) ;$ IR (film): $1493,1269 \mathrm{~cm}^{-1} ;{ }^{1} \mathrm{H}$ NMR $\left(400 \mathrm{MHz}, \mathrm{CDCl}_{3}\right) \delta 6.96(\mathrm{dd}, J=5.2,3.6,1 \mathrm{H}), 6.89-6.87(\mathrm{~m}, 2 \mathrm{H}), 3.89(\mathrm{~d}, J=4.6 \mathrm{~Hz}, 1 \mathrm{H})$, 
$3.82(\mathrm{~s}, 3 \mathrm{H}), 3.49(\mathrm{~d}, J=4.6 \mathrm{~Hz}, 1 \mathrm{H}), 1.65(\mathrm{~s}, 3 \mathrm{H}), 1.27(\mathrm{~s}, 3 \mathrm{H}) ;{ }^{13} \mathrm{C} \mathrm{NMR}\left(100 \mathrm{MHz}, \mathrm{CDCl}_{3}\right) \delta$ 149.3, 141.8, 121.7, 121.0, 120.9, 113.4, 73.4, 63.0, 56.4, 51.0, 25.9, 22.6; Anal. Calcd for $\mathrm{C}_{12} \mathrm{H}_{14} \mathrm{O}_{3}$ : C, 69.88; H, 6.84. Found: C, 69.60; H, 7.02.

\section{Table 1, entry 13}

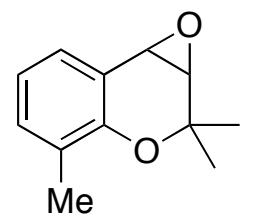

White solid; $\mathrm{mp} 37-38{ }^{\circ} \mathrm{C} ;[\alpha]^{20}{ }_{\mathrm{D}}=+12.0\left(c, 0.67, \mathrm{CHCl}_{3}\right)\left(81 \%\right.$ ee); IR (film): $1473 \mathrm{~cm}^{-1} ;{ }^{1} \mathrm{H}$ NMR (400 MHz, $\left.\mathrm{CDCl}_{3}\right) \delta 7.19(\mathrm{dd}, J=7.2,1.6 \mathrm{~Hz}, 1 \mathrm{H}), 7.12(\mathrm{dd}, J=8.0,1.2 \mathrm{~Hz}, 1 \mathrm{H}), 6.84(\mathrm{t}$, $J=7.6 \mathrm{~Hz}, 1 \mathrm{H}), 3.91(\mathrm{~d}, J=4.6 \mathrm{~Hz}, 1 \mathrm{H}), 3.51(\mathrm{~d}, J=4.6 \mathrm{~Hz}, 1 \mathrm{H}), 2.17(\mathrm{~s}, 3 \mathrm{H}), 1.61(\mathrm{~s}, 3 \mathrm{H})$, $1.24(\mathrm{~s}, 3 \mathrm{H}) ;{ }^{13} \mathrm{C} \mathrm{NMR}\left(100 \mathrm{MHz}, \mathrm{CDCl}_{3}\right) \delta 150.7,131.8,127.4,120.7,119.5,73.1,63.1,51.5$, 25.9, 22.9, 15.7. Anal. Calcd for $\mathrm{C}_{12} \mathrm{H}_{14} \mathrm{O}_{2}$ : C, 75.76; H, 7.42. Found: 75.60; H, 7.60.

\section{Table 1, entry 14}

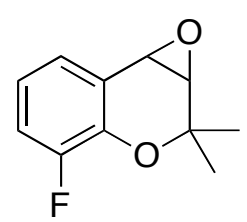

Yellow oil; $[\alpha]^{20}{ }_{D}=+13.1\left(c, 0.53, \mathrm{CHCl}_{3}\right)(84 \%$ ee $) ; \quad$ IR (film): $1491 \mathrm{~cm}^{-1} ;{ }^{1} \mathrm{H}$ NMR $(400$ $\left.\mathrm{MHz}, \mathrm{CDCl}_{3}\right) \delta 7.13(\mathrm{dd}, J=7.6,1.2 \mathrm{~Hz}, 1 \mathrm{H}), 7.08-7.03(\mathrm{~m}, 1 \mathrm{H}), 6.85(\mathrm{td}, J=7.6,4.4 \mathrm{~Hz}, 1 \mathrm{H})$, $3.93(\mathrm{dd}, J=4.4,1.6 \mathrm{~Hz}, 1 \mathrm{H}), 3.52(\mathrm{~d}, J=4.4 \mathrm{~Hz}, 1 \mathrm{H}), 1.64(\mathrm{~s}, 3 \mathrm{H}), 1.29(\mathrm{~s}, 3 \mathrm{H}) ;{ }^{13} \mathrm{C} \mathrm{NMR}$ $\left(100 \mathrm{MHz}, \mathrm{CDCl}_{3}\right) \delta 153.4,150.9,140.6,140.5,124.9,124.8,122.7,120.9,120.8,117.4,117.2$, 74.0, 62.8, 50.6, 25.6, 22.7; Anal. Calcd for $\mathrm{C}_{11} \mathrm{H}_{11} \mathrm{FO}_{2}$ : C, 68.03; H, 5.71. Found: C, 68.15; H, 5.90. 


\section{Table 1, entry 15}

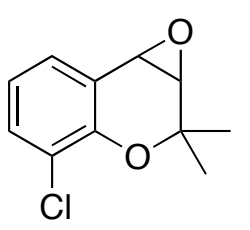

Yellow solid; mp $42-44{ }^{\circ} \mathrm{C} ;[\alpha]^{20}{ }_{D}=+30.8\left(c, 0.57, \mathrm{CHCl}_{3}\right)\left(83 \%\right.$ ee); IR (film): $1459 \mathrm{~cm}^{-1} ;{ }^{1} \mathrm{H}$ NMR $\left(400 \mathrm{MHz}, \mathrm{CDCl}_{3}\right) \delta 7.32(\mathrm{dd}, J=7.6,1.6 \mathrm{~Hz}, 1 \mathrm{H}), 7.25(\mathrm{dd}, J=7.6,1.4 \mathrm{~Hz}, 1 \mathrm{H}), 6.86(\mathrm{t}$, $J=7.6 \mathrm{~Hz}, 1 \mathrm{H}), 3.91(\mathrm{~d}, J=4.4 \mathrm{~Hz}, 1 \mathrm{H}), 3.52(\mathrm{~d}, J=4.4 \mathrm{~Hz}, 1 \mathrm{H}), 1.65(\mathrm{~s}, 3 \mathrm{H}), 1.27(\mathrm{~s}, 3 \mathrm{H}) ;{ }^{13} \mathrm{C}$ NMR $\left(100 \mathrm{MHz}, \mathrm{CDCl}_{3}\right) \delta 148.5,131.0,128.2,123.1,121.9,121.4,74.4,62.8,50.9,25.7,22.9$; Anal. Calcd for $\mathrm{C}_{11} \mathrm{H}_{11} \mathrm{ClO}_{2}$ : C, 62.72; H, 5.26. Found: C, 62.90; H, 5.28.

\section{Table 1, entry 16}

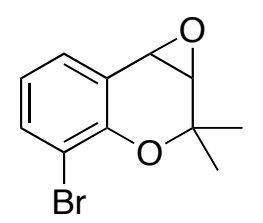

Yellow oil; $[\alpha]^{20}{ }_{D}=+45.3\left(c, 0.57, \mathrm{CHCl}_{3}\right)\left(82 \%\right.$ ee); IR (film): $1455 \mathrm{~cm}^{-1} ;{ }^{1} \mathrm{H}$ NMR $(400$ $\left.\mathrm{MHz}, \mathrm{CDCl}_{3}\right) \delta 7.50(\mathrm{dd}, J=8.4,1.6 \mathrm{~Hz}, 1 \mathrm{H}), 7.30(\mathrm{dd}, J=7.6,1.6 \mathrm{~Hz}, 1 \mathrm{H}), 6.82(\mathrm{dd}, J=8.0$, $7.6 \mathrm{~Hz}, 1 \mathrm{H}), 3.90(\mathrm{~d}, J=4.4 \mathrm{~Hz}, 1 \mathrm{H}), 3.52(\mathrm{~d}, J=4.4 \mathrm{~Hz}, 1 \mathrm{H}), 1.66(\mathrm{~s}, 3 \mathrm{H}), 1.27(\mathrm{~s}, 3 \mathrm{H}) ;{ }^{13} \mathrm{C}$ NMR $\left(100 \mathrm{MHz}, \mathrm{CDCl}_{3}\right) \delta 149.5,134.0,129.0,122.0,121.8,112.5,74.6,62.9,51.0,25.7,22.9$; Anal. Calcd for $\mathrm{C}_{11} \mathrm{H}_{11} \mathrm{BrO}_{2}$ : C, 51.79; H, 4.35. Found: C, 51.54; H, 4.39.

\section{Table 1, entry 17}<smiles>CC1(C)Oc2c(C#N)cccc2C2OC21</smiles> 
Yellow solid; $\mathrm{mp} 86-88^{\circ} \mathrm{C} ;[\alpha]^{20}{ }_{\mathrm{D}}=+88.7\left(c, 0.71, \mathrm{CHCl}_{3}\right)(88 \%$ ee); IR (film): 2229, 1458 $\mathrm{cm}^{-1} ;{ }^{1} \mathrm{H}$ NMR $\left(400 \mathrm{MHz}, \mathrm{CDCl}_{3}\right) \delta 7.56(\mathrm{dd}, J=7.6,2.0 \mathrm{~Hz}, 1 \mathrm{H}), 7.50(\mathrm{dd}, J=8.0,1.6 \mathrm{~Hz}$, 1H), $7.00(\mathrm{dd}, J=8.0,7.6 \mathrm{~Hz}, 1 \mathrm{H}), 3.93(\mathrm{~d}, J=4.4 \mathrm{~Hz}, 1 \mathrm{H}), 3.56(\mathrm{~d}, J=4.4 \mathrm{~Hz}, 1 \mathrm{H}), 1.64(\mathrm{~s}$, 3H), $1.33(\mathrm{~s}, 3 \mathrm{H}) ;{ }^{13} \mathrm{C} \mathrm{NMR}\left(100 \mathrm{MHz}, \mathrm{CDCl}_{3}\right) \delta 155.1,134.3,134.0,121.4,121.2,115.9$, 102.5, 75.6, 62.4, 50.1, 25.5, 23.1. HRMS calcd for $\mathrm{C}_{12} \mathrm{H}_{12} \mathrm{NO}_{2}(\mathrm{M}+1) 202.0868$, found 202.0867 . 
The chromatograms for the determination of enantiomeric excess of the epoxides from the asymmetric epoxidation of chromenes catalyzed by ketone 4 .

(3R, 4R)-2, 2-dimethyl-chromene oxide (Table 1, Entry 1)<smiles>CC1(C)Oc2ccccc2C2OC21</smiles>

HPLC Cond.: Column: Chiralcel OD (Column No. OD00CE-DL010), Chiral Technologies, Inc. Eluent: Hexanes/IPA (90/10); Flow Rate: 1.0mL/min; Detection: UV270
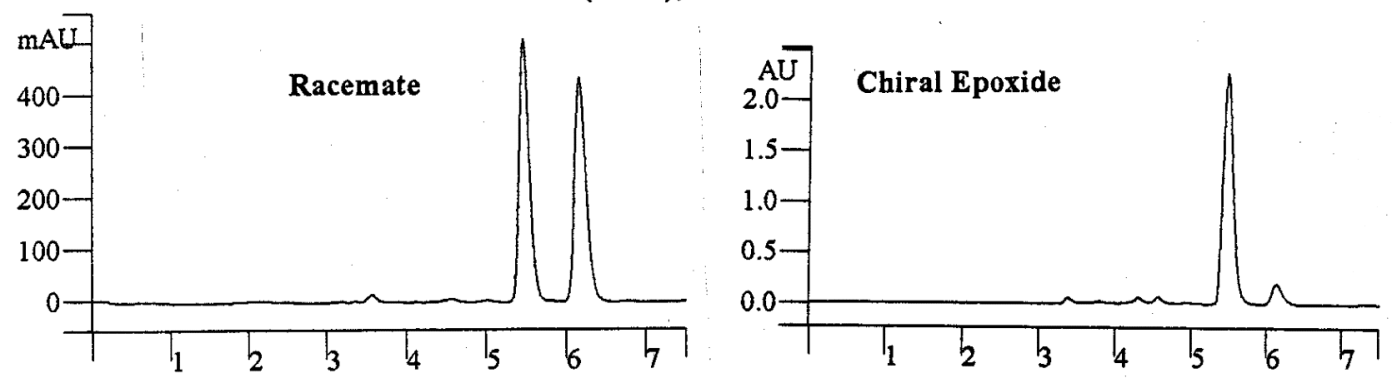

\begin{tabular}{|c|c|c|c|c|c|c|c|}
\hline \multirow[b]{2}{*}{$\begin{array}{c}\text { Peak } \\
\text { No }\end{array}$} & \multicolumn{5}{|c|}{ Minutes } & \multicolumn{2}{|c|}{ Minute } \\
\hline & $\begin{array}{c}\text { Peak } \\
\text { Name }\end{array}$ & $\begin{array}{l}\text { Ret. } \\
\text { Time } \\
\text { (min) }\end{array}$ & Result 0 & $\begin{array}{c}\text { Peak } \\
\text { No }\end{array}$ & $\begin{array}{l}\text { Peak } \\
\text { Name }\end{array}$ & $\begin{array}{l}\text { Ret. Time } \\
\quad \text { (min) }\end{array}$ & Result 0 \\
\hline 1 & & 5.471 & 50.1943 & 1 & & 5.456 & 91.7736 \\
\hline 2 & & 6.168 & 49.8057 & 2 & & 6.134 & 8.2264 \\
\hline & Totals & & 100.0000 & & Totals & & 100.0000 \\
\hline
\end{tabular}

2, 2-dimethyl-6-methoxy-chromene oxide (Table 1, Entry 2)<smiles>COc1ccc2c(c1)C1OC1C(C)(C)O2</smiles>

HPLC Cond: Column: Chiralcel OD (Column No. OD00CE-DL010), Chiral Technologies, Inc. Eluent: Hexanes/IPA (90/10); Flow Rate: $1.0 \mathrm{~mL} / \mathrm{min}$; Detection: UV220
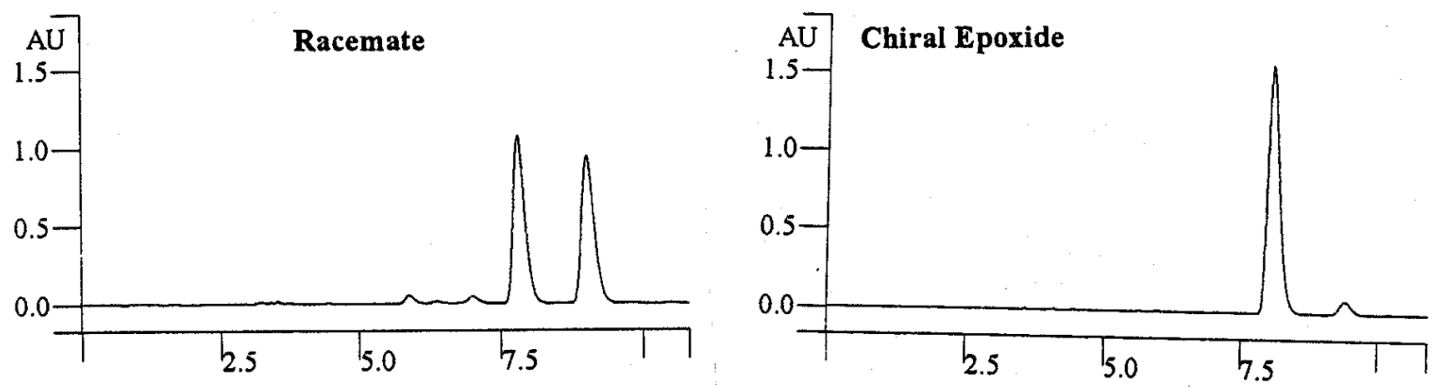

\begin{tabular}{|c|c|c|c|}
\hline $\begin{array}{c}\text { Peak } \\
\text { No }\end{array}$ & $\begin{array}{l}\text { Peak } \\
\text { Name }\end{array}$ & $\begin{array}{l}\text { Ret. } \\
\text { Time } \\
\text { (min) }\end{array}$ & $\begin{array}{l}\text { Minutes } \\
\text { Result } 0\end{array}$ \\
\hline $\begin{array}{l}1 \\
2\end{array}$ & & $\begin{array}{l}7.796 \\
8.996 \\
\end{array}$ & $\begin{array}{r}49.9988 \\
50.0012 \\
\end{array}$ \\
\hline & Totals & & 100.0000 \\
\hline
\end{tabular}

\begin{tabular}{cccc}
$\begin{array}{c}\text { Peak } \\
\text { No }\end{array}$ & $\begin{array}{c}\text { Peak } \\
\text { Name }\end{array}$ & $\begin{array}{c}\text { Ret. } \\
\text { Time } \\
(\mathbf{m i n})\end{array}$ & $\begin{array}{c}\text { Minutes } \\
\text { Result } 0\end{array}$ \\
\hline$\frac{2}{8.031}$ & $\frac{95.0277}{\text { Totals }}$ & $\frac{9.392}{4.9723}$ \\
\hline
\end{tabular}


2, 2-dimethyl-6-methyl-chromene oxide (Table 1, Entry 3)

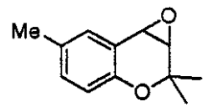

HPLC Cond.: Column: Chiralcel OD (Column No. OD00CE-DL010), Chiral Technologies, Inc.

Eluent: Hexanes/IPA (90/10); Flow Rate: $1.0 \mathrm{~mL} / \mathrm{min}$; Detection: UV270
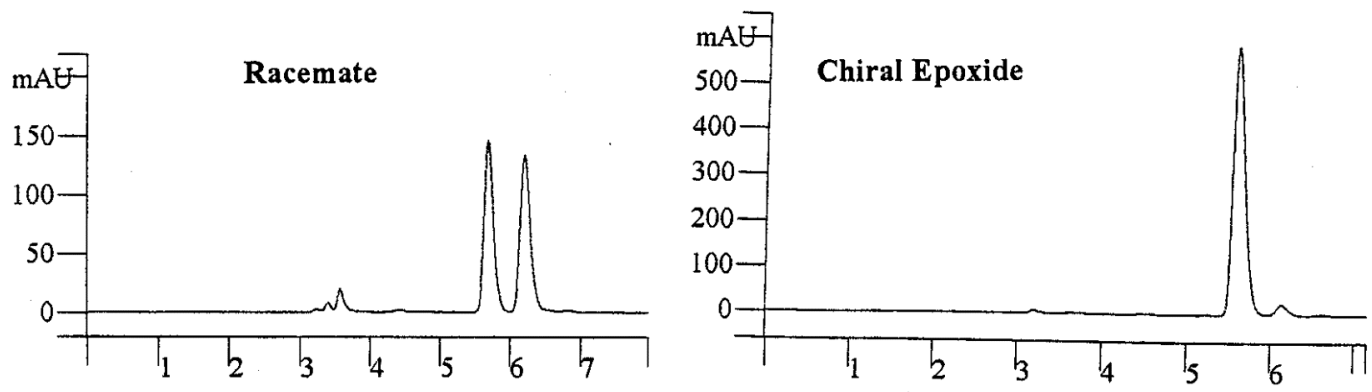

\begin{tabular}{|c|c|c|c|}
\hline $\begin{array}{c}\text { Peak } \\
\text { No }\end{array}$ & $\begin{array}{l}\text { Peak } \\
\text { Name }\end{array}$ & $\begin{array}{l}\text { Ret. Time } \\
\text { (min) }\end{array}$ & $\begin{array}{l}\text { Minut } \\
\text { Result } 0\end{array}$ \\
\hline $\begin{array}{l}1 \\
2\end{array}$ & & $\begin{array}{l}5.686 \\
6.201\end{array}$ & $\begin{array}{l}50.1994 \\
49.8006 \\
\end{array}$ \\
\hline & Tota & & 100.0000 \\
\hline
\end{tabular}

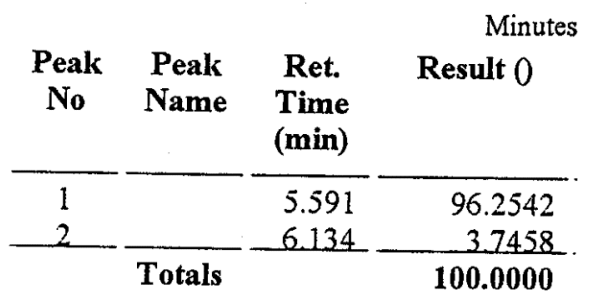

2, 2-dimethyl-6-ethyl-chromene oxide (Table 1, Entry 4)

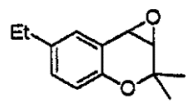

HPLC Cond: Column: Chiralcel OB (Column No. OB00CE-GJ019), Chiral Technologies, Inc.

Eluent: Hexanes/IPA (90/10); Flow Rate: 1.0mL/min; Detection: UV220

Racemate

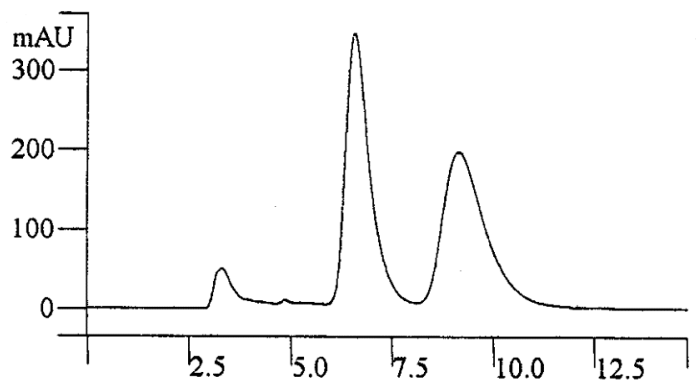

Minutes

\begin{tabular}{|c|c|c|c|}
\hline $\begin{array}{c}\text { Peak } \\
\text { No }\end{array}$ & $\begin{array}{l}\text { Peak } \\
\text { Name }\end{array}$ & $\begin{array}{l}\text { Ret. } \\
\text { Time } \\
\text { (min) }\end{array}$ & Result 0 \\
\hline 1 & & 6.526 & $\begin{array}{l}50.0766 \\
499234\end{array}$ \\
\hline & Totals & 9.109 & $\begin{array}{r}49.9234 \\
100.0000\end{array}$ \\
\hline
\end{tabular}

Chiral Epoxide

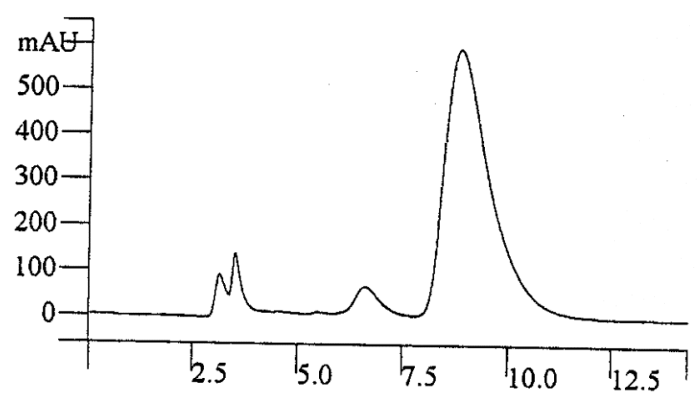

Minutes

\begin{tabular}{|c|c|c|c|}
\hline $\begin{array}{c}\text { Peak } \\
\text { No }\end{array}$ & $\begin{array}{l}\text { Peak } \\
\text { Name }\end{array}$ & $\begin{array}{l}\text { Ret. } \\
\text { Time } \\
\text { (min) }\end{array}$ & Result 0 \\
\hline $\begin{array}{l}1 \\
2 \\
\end{array}$ & & $\begin{array}{l}6.596 \\
8.799\end{array}$ & $\begin{array}{r}5.1937 \\
94.8063\end{array}$ \\
\hline & Totals & & 100.0000 \\
\hline
\end{tabular}


2, 2-dimethyl-6-n-propyl-chromene oxide (Table 1, Entry 5)<smiles>CC1(C)Oc2ccc([18F])cc2O1</smiles>

HPLC Cond.: Column: Chiralcel OD (Column No. OD00CE-DL010), Chiral Technologies, Inc. Eluent: Hexanes/IPA (90/10); Flow Rate: $1.0 \mathrm{~mL} / \mathrm{min}$; Detection: UV270
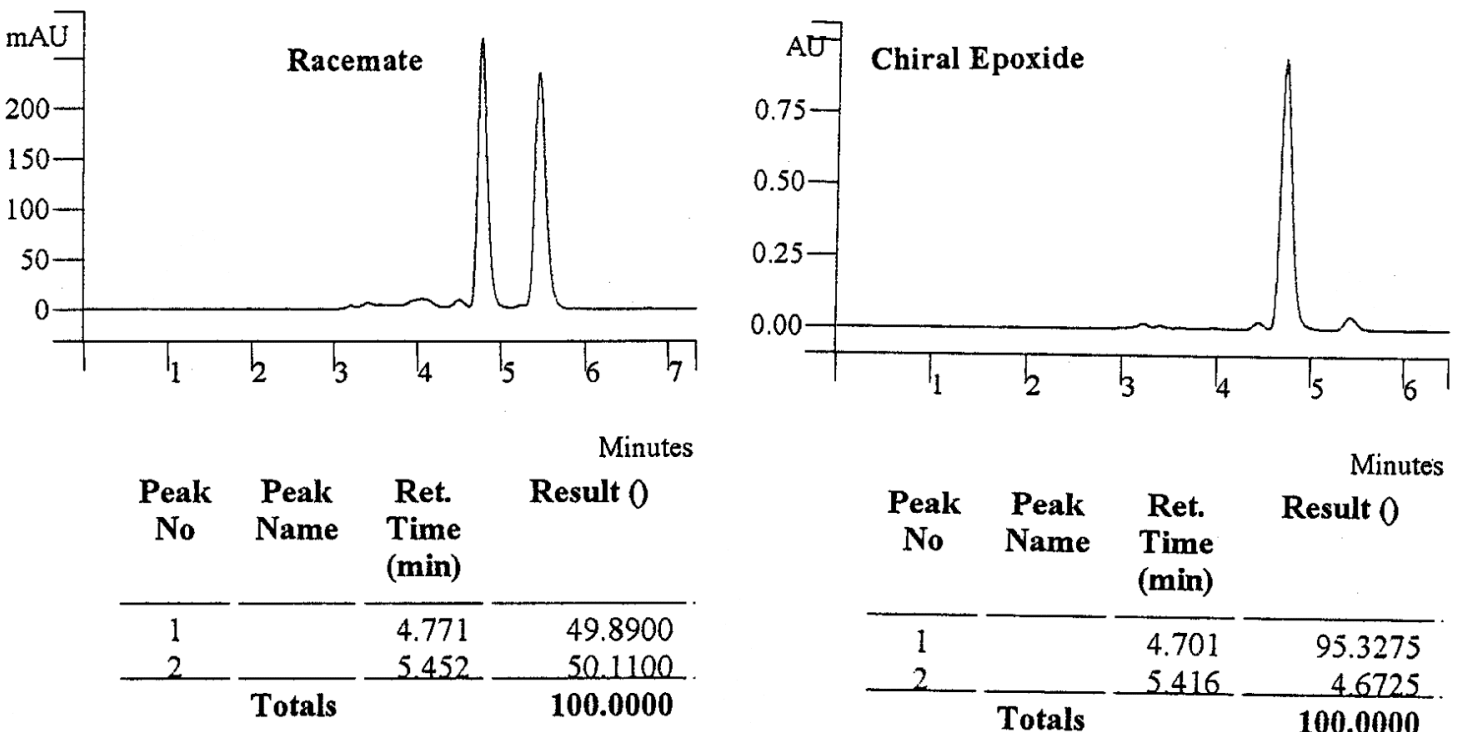

\begin{tabular}{|c|c|c|c|}
\hline $\begin{array}{c}\text { Peak } \\
\text { No }\end{array}$ & $\begin{array}{c}\text { Peak } \\
\text { Name }\end{array}$ & $\begin{array}{l}\text { Ret. } \\
\text { Time } \\
\text { (min) }\end{array}$ & $\begin{array}{l}\text { Minutes } \\
\text { Result } 0\end{array}$ \\
\hline $\begin{array}{l}1 \\
2 \\
\end{array}$ & & $\begin{array}{l}4.701 \\
5416 \\
\end{array}$ & $\begin{array}{r}95.3275 \\
4.6725 \\
\end{array}$ \\
\hline & Totals & & 100.0000 \\
\hline
\end{tabular}

2, 2-dimethyl-6-i-propyl -chromene oxide (Table 1, Entry 6)<smiles>CC1(C)Oc2ccc([PH2+])cc2O1</smiles>

HPLC Cond.: Column: Chiralcel OD (Column No. OD00CE-DL010), Chiral Technologies, Inc.

Eluent: Hexanes/IPA (90/10); Flow Rate: $1.0 \mathrm{~mL} / \mathrm{min}$; Detection: UV270
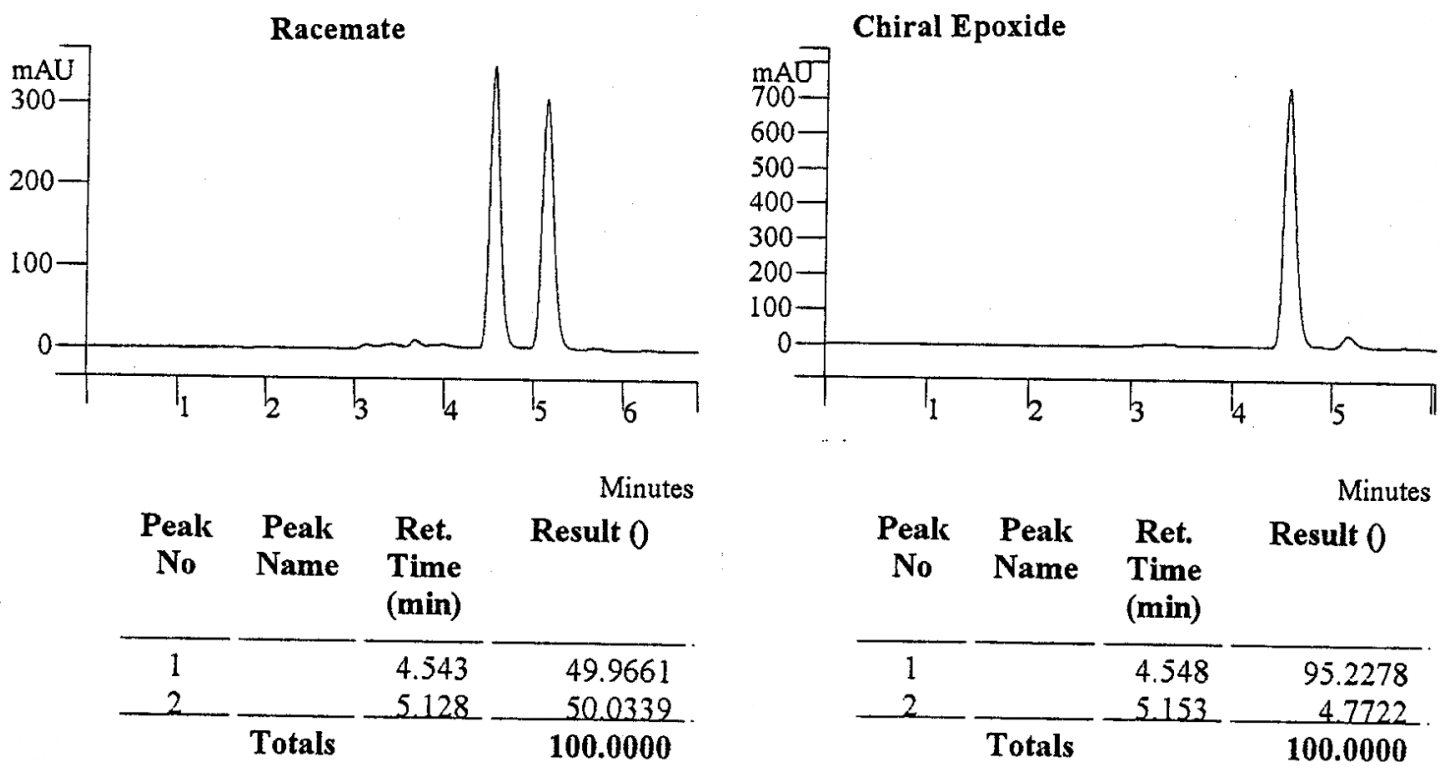
2, 2-dimethyl-6-fluoro-chromene oxide (Table 1, Entry 7)<smiles>CC1(C)Oc2ccc(F)cc2C2OC21</smiles>

GC Cond.:
Column: Chiraldex BDM (Cat. No. 77023), Adv. Separation Technologies, Inc Oven: $125^{\circ} \mathrm{C}$; Carrier: Helium, head pressure 30 psi; Detection: FID $250^{\circ} \mathrm{C}$
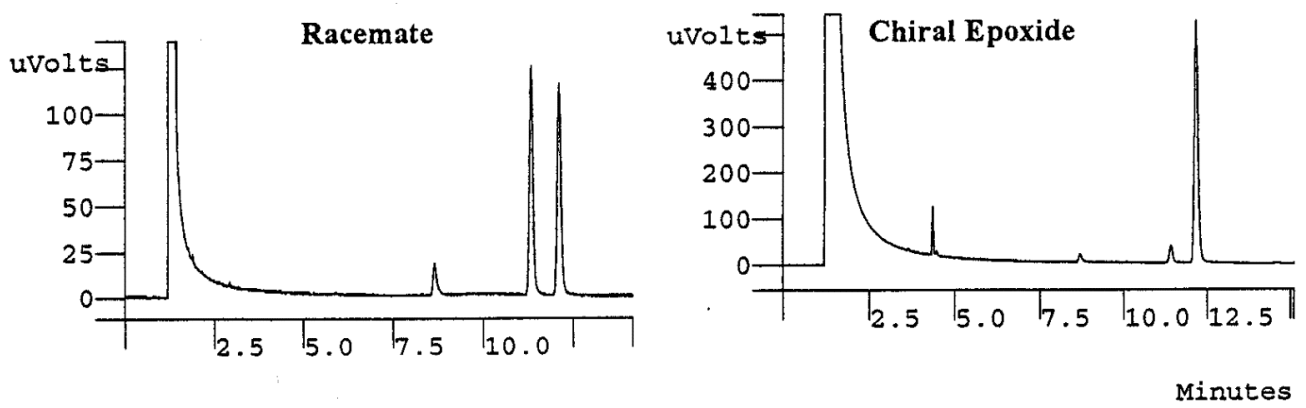

\begin{tabular}{|c|c|c|c|}
\hline $\begin{array}{c}\text { Peak } \\
\text { No }\end{array}$ & $\begin{array}{c}\text { Peak } \\
\text { Name }\end{array}$ & $\begin{array}{c}\text { Ret. Time } \\
(\min )\end{array}$ & $\begin{array}{l}\text { Minutes } \\
\text { Result } 0\end{array}$ \\
\hline 1 & & 11.318 & 50.2098 \\
\hline 2 & & 12.092 & 49.7902 \\
\hline & Totals & & 100.0000 \\
\hline
\end{tabular}

\begin{tabular}{|c|c|c|c|}
\hline $\begin{array}{c}\text { Peak } \\
\text { No }\end{array}$ & $\begin{array}{c}\text { Peak } \\
\text { Name }\end{array}$ & $\begin{array}{l}\text { Ret. } \\
\text { Time } \\
\text { (min) }\end{array}$ & Result 0 \\
\hline 1 & & 11.399 & 5.6647 \\
\hline 2 & & 12.131 & 94.3353 \\
\hline
\end{tabular}

2, 2-dimethyl-6-chloro-chromene oxide (Table 1, Entry 8)<smiles>CC1(C)Oc2ccc(Cl)cc2C2OC21</smiles>

HPLC Cond.: Column: Chiralcel OD (Column No. OD00CE-DL010), Chiral Technologies, Inc. Eluent: Hexanes/IPA (90/10); Flow Rate: $1.0 \mathrm{~mL} / \mathrm{min}$; Detection: UV220

Racemate

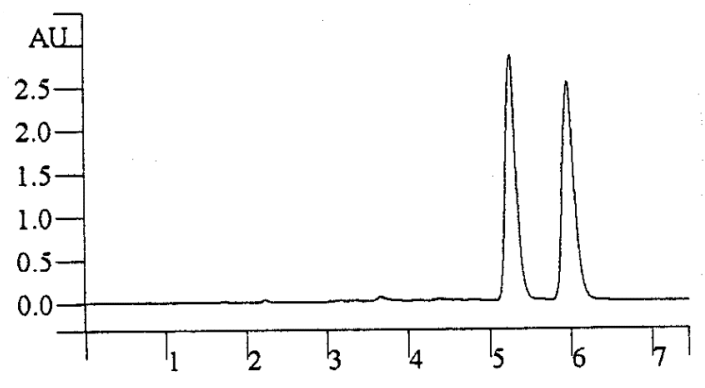

Minutes

\begin{tabular}{|c|c|c|c|}
\hline $\begin{array}{c}\text { Peak } \\
\text { No }\end{array}$ & $\begin{array}{l}\text { Peak } \\
\text { Name }\end{array}$ & $\begin{array}{l}\text { Ret. } \\
\text { Time } \\
\text { (min) }\end{array}$ & Result 0 \\
\hline $\begin{array}{l}1 \\
2\end{array}$ & & $\begin{array}{l}5.261 \\
5.961\end{array}$ & $\begin{array}{l}49.8101 \\
50.1899\end{array}$ \\
\hline & Totals & & 100.0000 \\
\hline
\end{tabular}

Chiral Epoxide

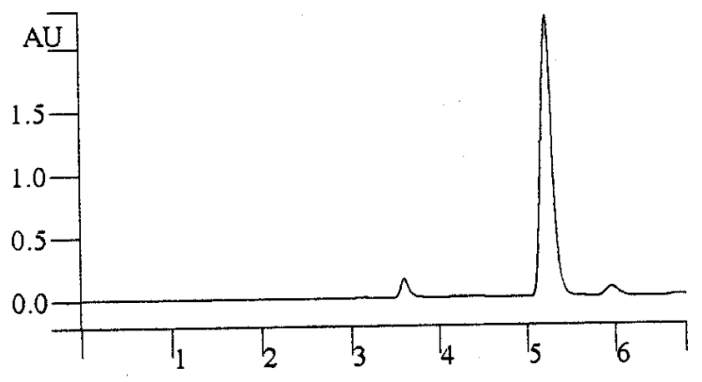

Minutes

\begin{tabular}{|c|c|c|c|}
\hline $\begin{array}{c}\text { Peak } \\
\text { No }\end{array}$ & $\begin{array}{l}\text { Peak } \\
\text { Name }\end{array}$ & $\begin{array}{l}\text { Ret. } \\
\text { Time } \\
\text { (min) }\end{array}$ & Result 0 \\
\hline $\begin{array}{l}1 \\
2\end{array}$ & & $\begin{array}{l}5.219 \\
5949\end{array}$ & $\begin{array}{r}96.5455 \\
34545\end{array}$ \\
\hline & Totals & & 100.0000 \\
\hline
\end{tabular}


2, 2-dimethyl-6-bromo-chromene oxide (Table 1, Entry 9)

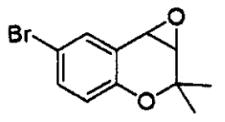

HPLC Cond: Column: Chiralcel OD (Column No. OD00CE-DL010), Chiral Technologies, Inc. Eluent: Hexanes/IPA (90/10); Flow Rate: 1.0mL/min; Detection: UV220
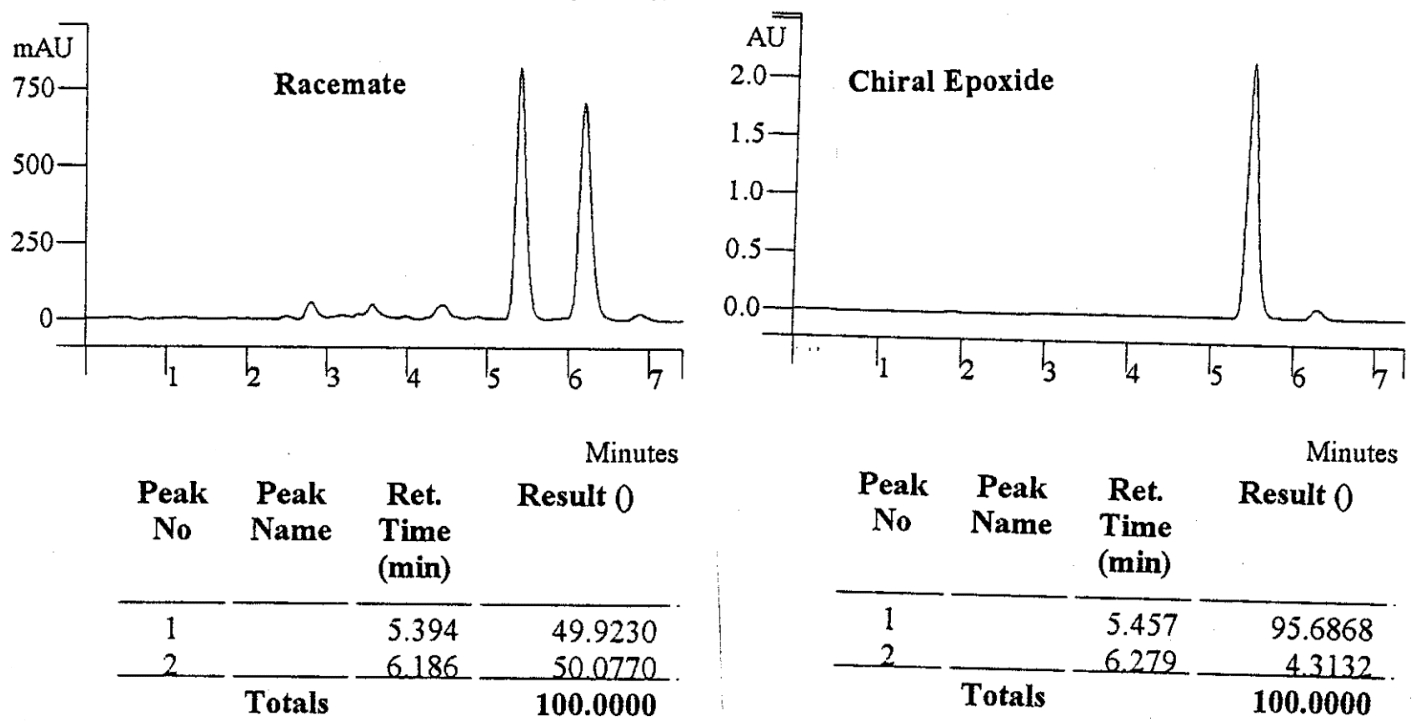

(3R, 4R)-2, 2-dimethyl-6-cyano-chromene oxide (Table 1, Entry 10)<smiles>CC1(C)Oc2ccc(C#N)cc2C2OC21</smiles>

HPLC Cond: Column: Chiralcel OD (Column No. OD00CE-DL010), Chiral Technologies, Inc. Eluent: Hexanes/IPA (90/10); Flow Rate: $0.6 \mathrm{~mL} / \mathrm{min}$; Detection: UV254
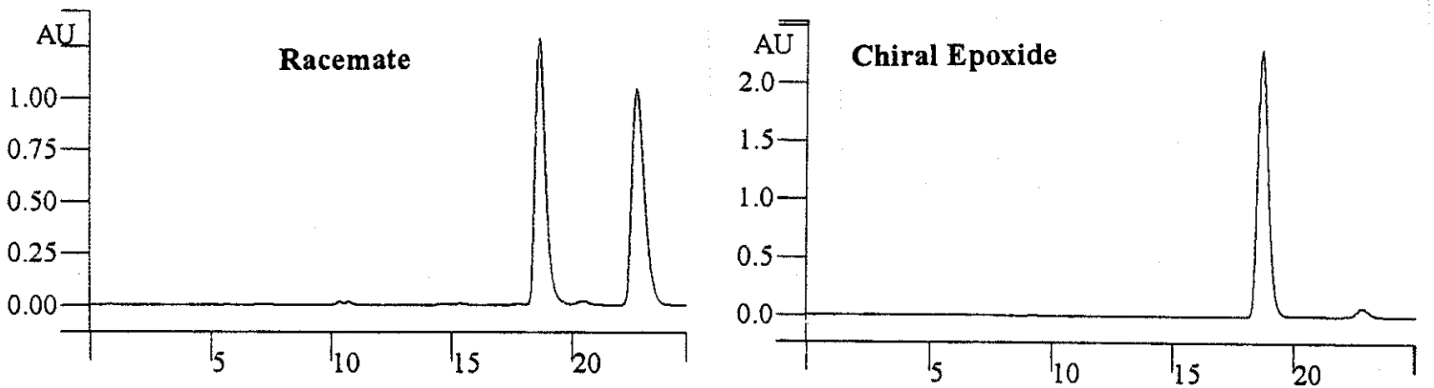

\begin{tabular}{|c|c|c|c|}
\hline $\begin{array}{c}\text { Peak } \\
\text { No }\end{array}$ & $\begin{array}{l}\text { Peak } \\
\text { Name }\end{array}$ & $\begin{array}{l}\text { Ret. } \\
\text { Time } \\
\text { (min) }\end{array}$ & $\begin{array}{l}\text { Minutes } \\
\text { Result } 0\end{array}$ \\
\hline $\begin{array}{l}1 \\
2\end{array}$ & & $\begin{array}{l}18.663 \\
22657\end{array}$ & 50.0780 \\
\hline & Totals & & 100.0000 \\
\hline
\end{tabular}

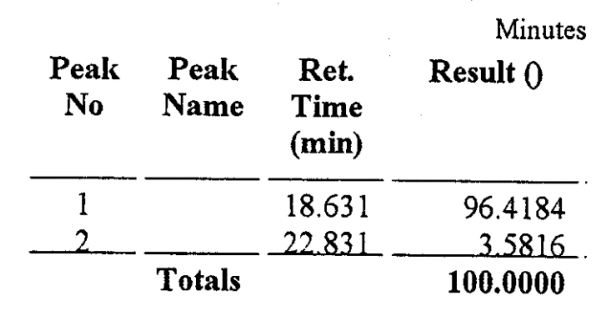


Me<smiles>c1ccc2c(c1)OCC1OC21</smiles>

HPLC Cond: Column: Chiralcel OD (Column No. OD00CE-DL010), Chiral Technologies, Inc.

Eluent: Hexanes/IPA (92/08); Flow Rate: $1.0 \mathrm{~mL} / \mathrm{min}$; Detection: UV220

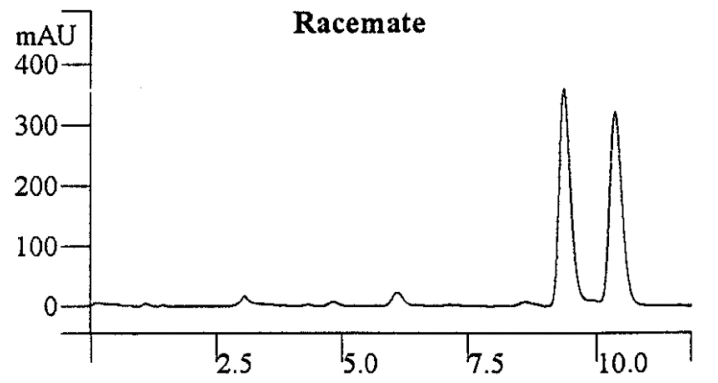

Minutes

\begin{tabular}{|c|c|c|c|}
\hline $\begin{array}{c}\text { Peak } \\
\text { No }\end{array}$ & $\begin{array}{c}\text { Peak } \\
\text { Name }\end{array}$ & $\begin{array}{l}\text { Ret. } \\
\text { Time } \\
\text { (min) }\end{array}$ & Result 0 \\
\hline 1 & & $\begin{array}{r}9.364 \\
10359\end{array}$ & $\begin{array}{l}50.0920 \\
499080\end{array}$ \\
\hline & at & & 100.0000 \\
\hline
\end{tabular}

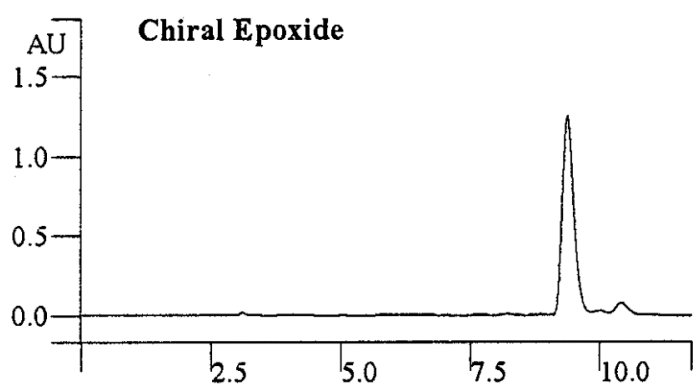

Minutes

\begin{tabular}{|c|c|c|c|}
\hline $\begin{array}{c}\text { Peak } \\
\text { No }\end{array}$ & $\begin{array}{c}\text { Peak } \\
\text { Name }\end{array}$ & $\begin{array}{l}\text { Ret. } \\
\text { Time } \\
\text { (min) }\end{array}$ & Result 0 \\
\hline $\begin{array}{l}1 \\
2\end{array}$ & & $\begin{array}{r}9.342 \\
10.421\end{array}$ & $\begin{array}{r}94.9354 \\
5.0646\end{array}$ \\
\hline & Totals & & 100.0000 \\
\hline
\end{tabular}

2, 2-dimethyl-8-methoxyl-chromene oxide (Table 1, Entry 12)

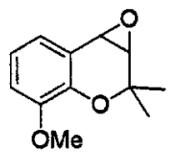

HPLC Cond.: Column: Chiralcel OJ (Column No. OJ00CE-DE008), Chiral Technologies, Inc.

Eluent: Hexanes/IPA (90/10); Flow Rate: $1.0 \mathrm{~mL} / \mathrm{min}$; Detection: UV220
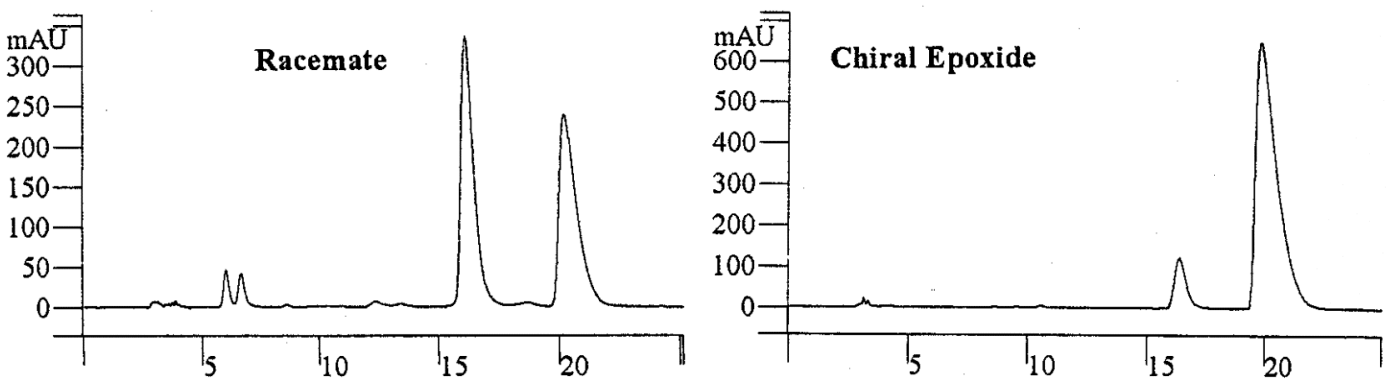

Minutes

\begin{tabular}{|c|c|c|c|}
\hline $\begin{array}{c}\text { Peak } \\
N_{0}\end{array}$ & $\begin{array}{l}\text { Peak } \\
\text { Name }\end{array}$ & $\begin{array}{l}\text { Ret. } \\
\text { Time } \\
\text { (min) }\end{array}$ & Result 0 \\
\hline $\begin{array}{l}1 \\
2\end{array}$ & & $\begin{array}{l}16.103 \\
20.179 \\
\end{array}$ & $\begin{array}{r}50.1315 \\
498685\end{array}$ \\
\hline
\end{tabular}

\begin{tabular}{|c|c|c|c|}
\hline $\begin{array}{c}\text { Peak } \\
\text { No }\end{array}$ & $\begin{array}{c}\text { Peak } \\
\text { Name }\end{array}$ & $\begin{array}{l}\text { Ret. } \\
\text { Time } \\
\text { (min) }\end{array}$ & $\begin{array}{l}\text { Minute } \\
\text { Result } 0\end{array}$ \\
\hline $\begin{array}{l}1 \\
2\end{array}$ & & 16.339 & 8.9701 \\
\hline 2 & Totals & 19.756 & $\frac{91.0299}{100.0000}$ \\
\hline
\end{tabular}


2, 2-dimethyl-8-methyl-chromene oxide (Table 1, Entry 13)

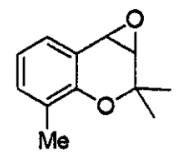

HPLC Cond: Column: Chiralcel OJ (Column No. OJ00CE-DE008), Chiral Technologies, Inc. Eluent: Hexanes/IPA (90/10); Flow Rate: 1.0mL/min; Detection: UV220
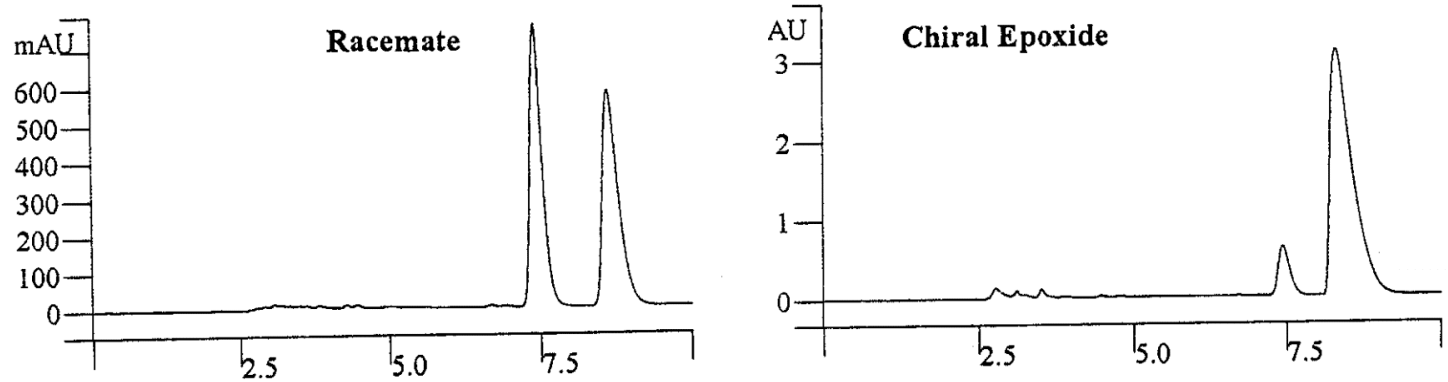

\begin{tabular}{|c|c|c|c|}
\hline $\begin{array}{c}\text { Peak } \\
\text { No }\end{array}$ & $\begin{array}{l}\text { Peak } \\
\text { Name }\end{array}$ & $\begin{array}{l}\text { Ret. } \\
\text { Time } \\
\text { (min) }\end{array}$ & $\begin{array}{l}\text { Minutes } \\
\text { Result } 0\end{array}$ \\
\hline $\begin{array}{l}1 \\
2\end{array}$ & & $\begin{array}{l}7.414 \\
8.618 \\
\end{array}$ & $\begin{array}{l}49.8073 \\
50.1927\end{array}$ \\
\hline & Total & & 100.0000 \\
\hline
\end{tabular}

\begin{tabular}{|c|c|c|c|}
\hline $\begin{array}{c}\text { Peak } \\
\text { No }\end{array}$ & $\begin{array}{l}\text { Peak } \\
\text { Name }\end{array}$ & $\begin{array}{l}\text { Ret. } \\
\text { Time } \\
\text { (min) }\end{array}$ & $\begin{array}{l}\text { Minutes } \\
\text { Result } 0\end{array}$ \\
\hline 1 & & 7.424 & 9.7313 \\
\hline & & 8.292 & $\frac{90.2081}{100.0000}$ \\
\hline
\end{tabular}

2,2-dimethyl-8-fluoro-chromene oxide (Table 1, Entry 14)<smiles>CC1(C)Oc2c(F)cccc2C2OC21</smiles>

HPLC Cond: Column: Chiralcel OJ (Column No. OJ00CE-DE008), Chiral Technologies, Inc. Eluent: Hexanes/IPA (90/10); Flow Rate: $1.0 \mathrm{~mL} / \mathrm{min}$; Detection: UV220
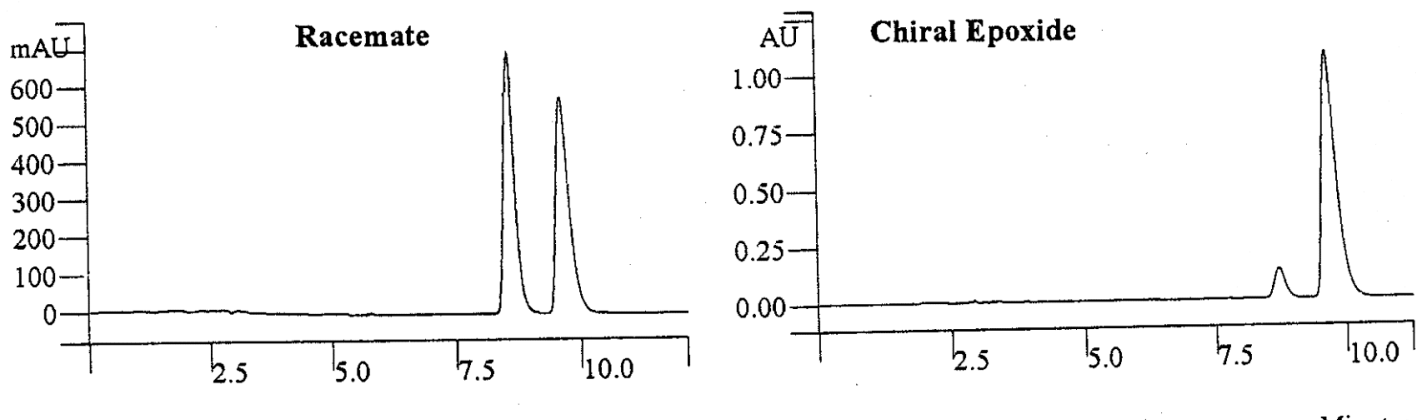

\begin{tabular}{|c|c|c|c|}
\hline $\begin{array}{c}\text { Peak } \\
\text { No }\end{array}$ & $\begin{array}{l}\text { Peak } \\
\text { Name }\end{array}$ & $\begin{array}{l}\text { Ret. } \\
\text { Time } \\
\text { (min) }\end{array}$ & $\begin{array}{l}\text { Minutes } \\
\text { Result } 0\end{array}$ \\
\hline $\begin{array}{l}1 \\
2\end{array}$ & & $\begin{array}{l}8.546 \\
9.601\end{array}$ & $\begin{array}{l}50.0898 \\
49.9102\end{array}$ \\
\hline & Totals & & 100.0000 \\
\hline
\end{tabular}

\begin{tabular}{|c|c|c|c|}
\hline $\begin{array}{c}\text { Peak } \\
\text { No }\end{array}$ & $\begin{array}{l}\text { Peak } \\
\text { Name }\end{array}$ & $\begin{array}{l}\text { Ret. } \\
\text { Time } \\
\text { (min) }\end{array}$ & $\begin{array}{l}\text { Minutes } \\
\text { Result } 0\end{array}$ \\
\hline 1 & & $\begin{array}{l}8.699 \\
9623\end{array}$ & $\begin{array}{r}8.0649 \\
919351\end{array}$ \\
\hline & Totals & & 100.0000 \\
\hline
\end{tabular}


<smiles>CC1(C)Oc2c(Cl)cccc2C2OC21</smiles>

HPLC Cond: Column: Chiralcel OJ (Column No. OJO0CE-DE008), Chiral Technologies, Inc.

Eluent: Hexanes/IPA (90/10); Flow Rate: $1.0 \mathrm{~mL} / \mathrm{min}$; Detection: UV220
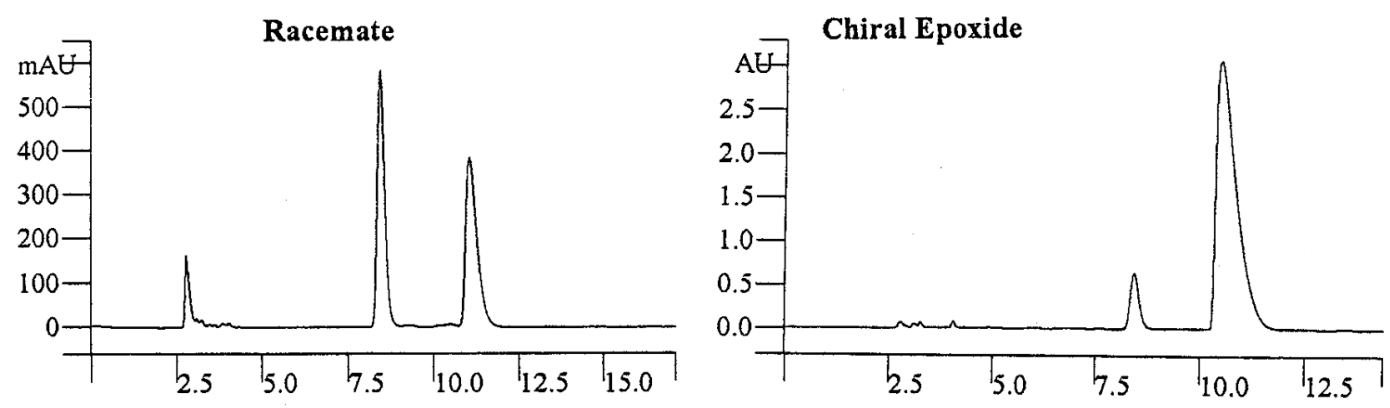

Minutes

\begin{tabular}{|c|c|c|c|}
\hline $\begin{array}{c}\text { Peak } \\
\text { No }\end{array}$ & $\begin{array}{l}\text { Peak } \\
\text { Name }\end{array}$ & $\begin{array}{l}\text { Ret. } \\
\text { Time } \\
\text { (min) }\end{array}$ & Result 0 \\
\hline $\begin{array}{l}1 \\
2\end{array}$ & & $\begin{array}{r}8.431 \\
11.042 \\
\end{array}$ & $\begin{array}{l}50.0751 \\
49.9249\end{array}$ \\
\hline
\end{tabular}

2,2-dimethyl-8-bromo-chromene oxide (Table 1, Entry 16)<smiles>CC1(C)Oc2c(Br)cccc2C2OC21</smiles>

HPLC Cond.: Column: Chiralcel OJ (Column No. OJ00CE-DE008), Chiral Technologies, Inc. Eluent: Hexanes/IPA (90/10); Flow Rate: $1.0 \mathrm{~mL} / \mathrm{min}$; Detection: UV270

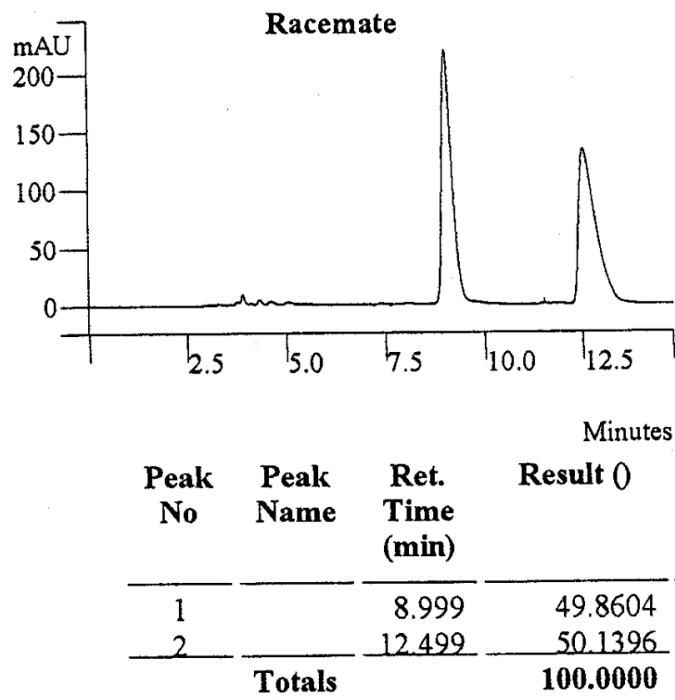

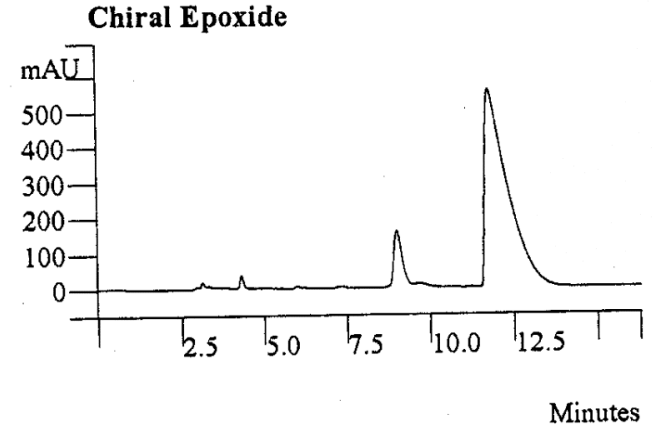

\begin{tabular}{|c|c|c|c|}
\hline $\begin{array}{c}\text { Peak } \\
\text { No }\end{array}$ & $\begin{array}{l}\text { Peak } \\
\text { Name }\end{array}$ & $\begin{array}{l}\text { Ret. Time } \\
\text { (min) }\end{array}$ & Result 0 \\
\hline 1 & & 8.988 & 9.0341 \\
\hline 2 & & 11.761 & 90.9659 \\
\hline & Totals & & 100.0000 \\
\hline
\end{tabular}


2, 2-dimethyl-8-cyano-chromene oxide (Table 1, Entry 17)

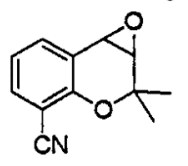

GC Cond.: Column: Chiraldex BDM (Cat. No. 77023), Adv. Separation Technologies, Inc. Oven: $130^{\circ} \mathrm{C}$; Carrier: Helium, head pressure 25 psi; Detection: $\mathrm{FD} 250^{\circ} \mathrm{C}$

Racemate
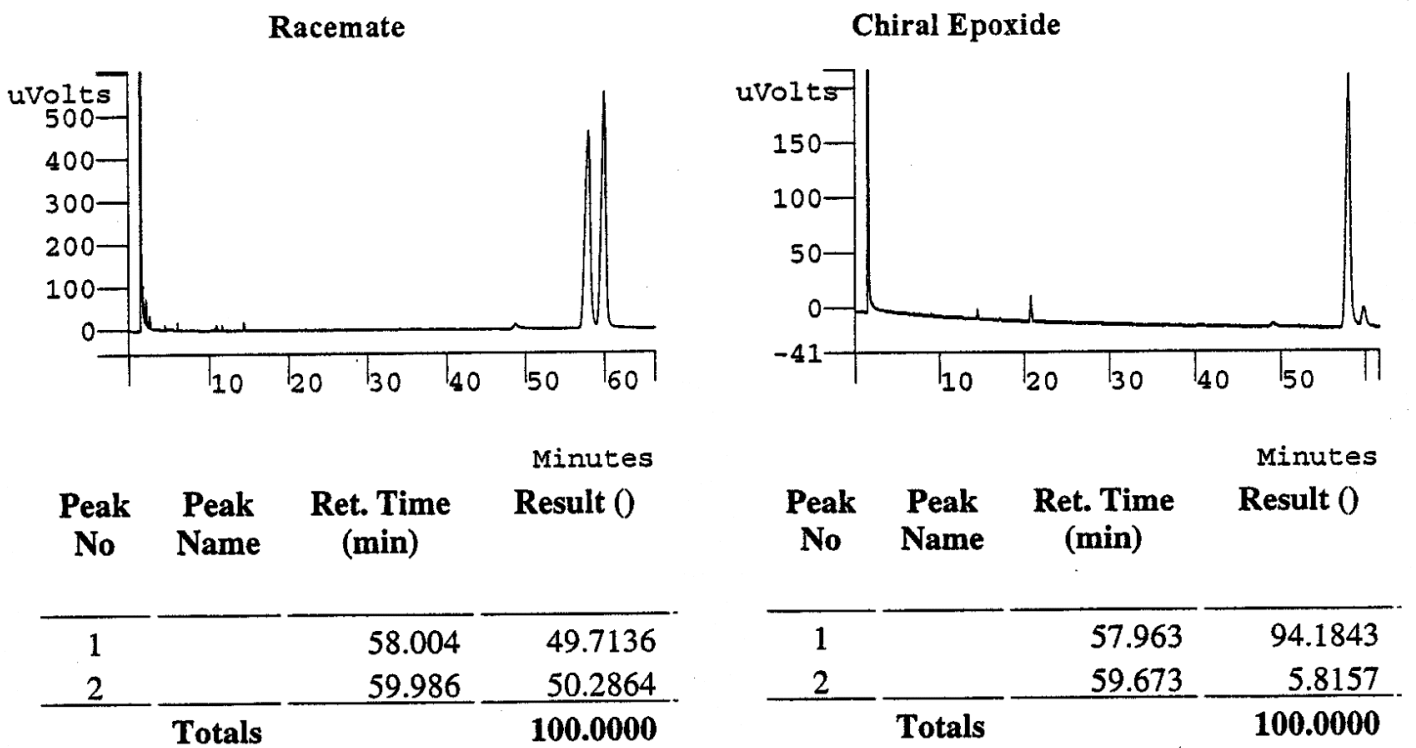

Minutes

\begin{tabular}{|c|c|c|c|}
\hline $\begin{array}{c}\text { Peak } \\
\text { No }\end{array}$ & $\begin{array}{l}\text { Peak } \\
\text { Name }\end{array}$ & $\begin{array}{l}\text { Ret. Time } \\
\text { (min) }\end{array}$ & Result () \\
\hline 1 & & 58.004 & 49.7136 \\
\hline 2 & & 59.986 & 50.2864 \\
\hline
\end{tabular}

Peak Peak Ret. Time Result ()

\begin{tabular}{|c|c|c|}
\hline 1 & 57.963 & 94.1843 \\
\hline 2 & 59.673 & 5.8157 \\
\hline
\end{tabular}



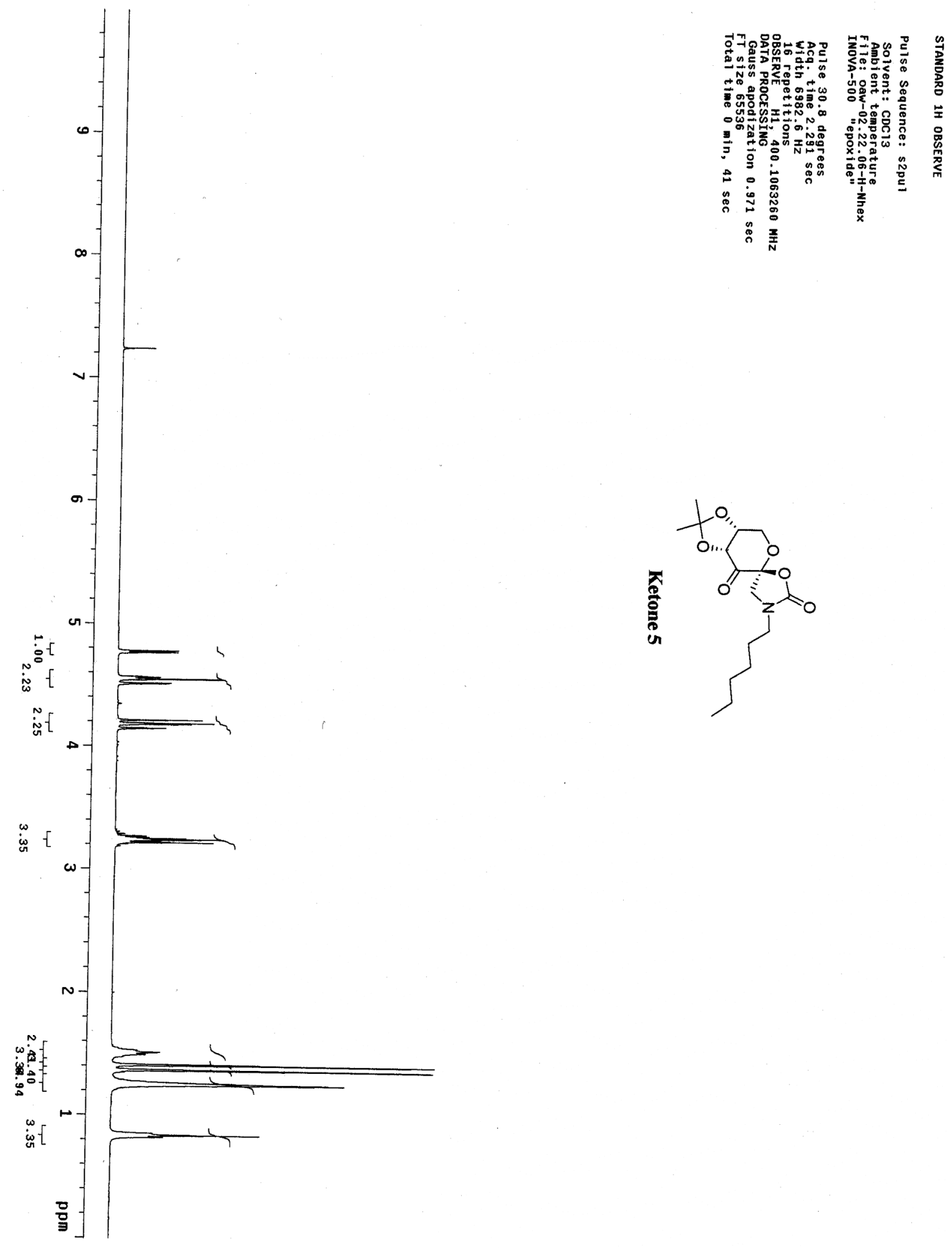

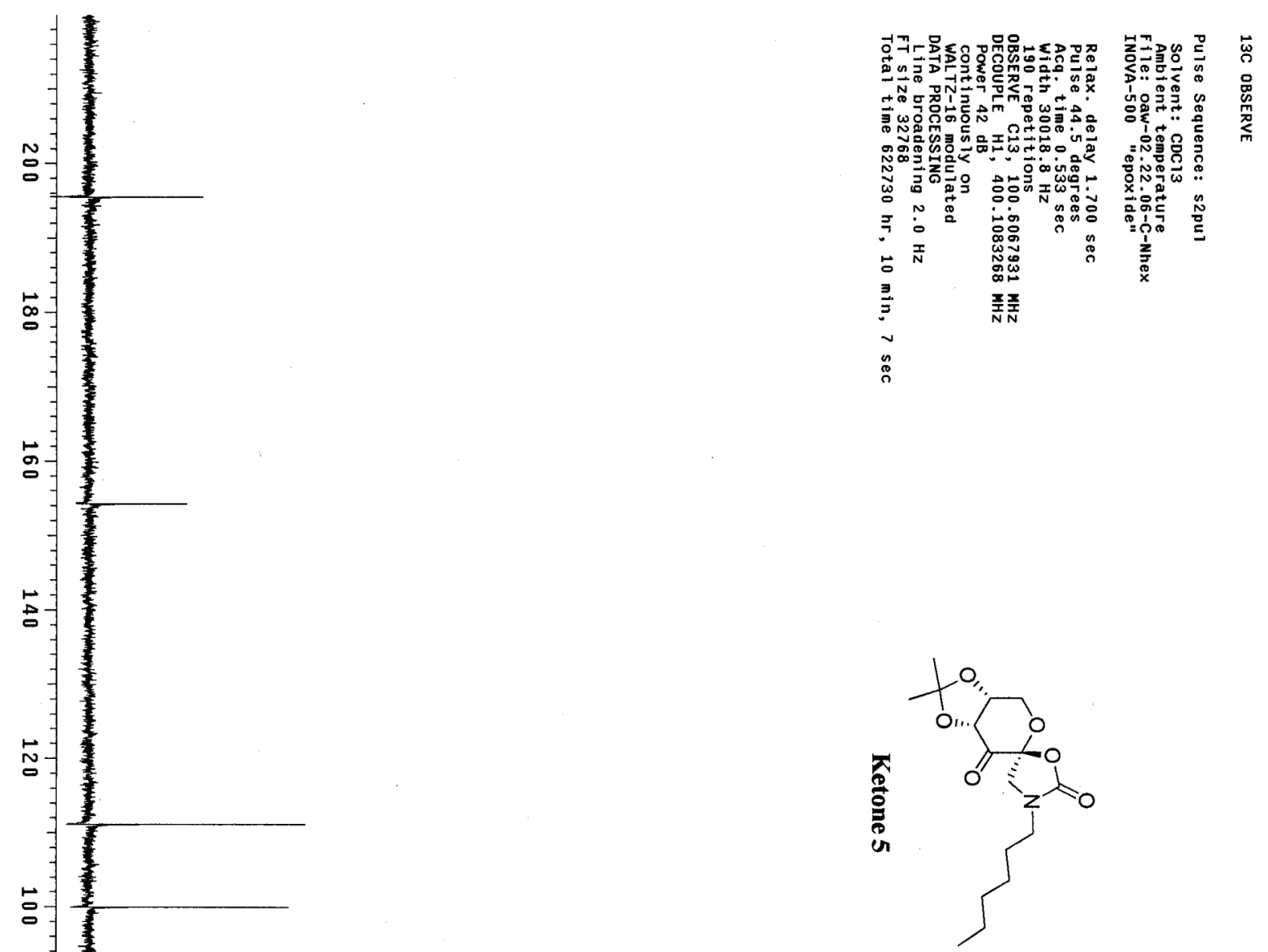

$\stackrel{\infty}{\infty}$

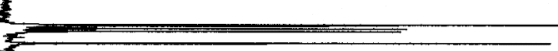

g-

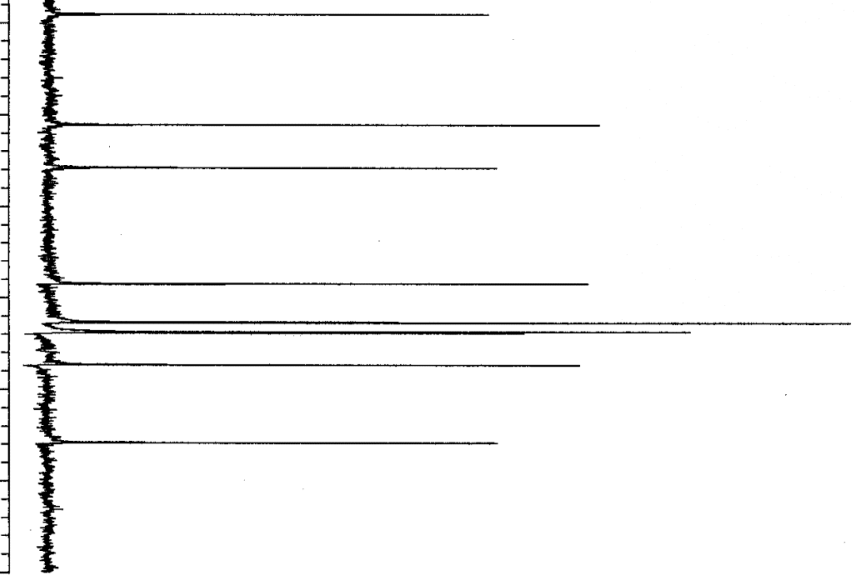



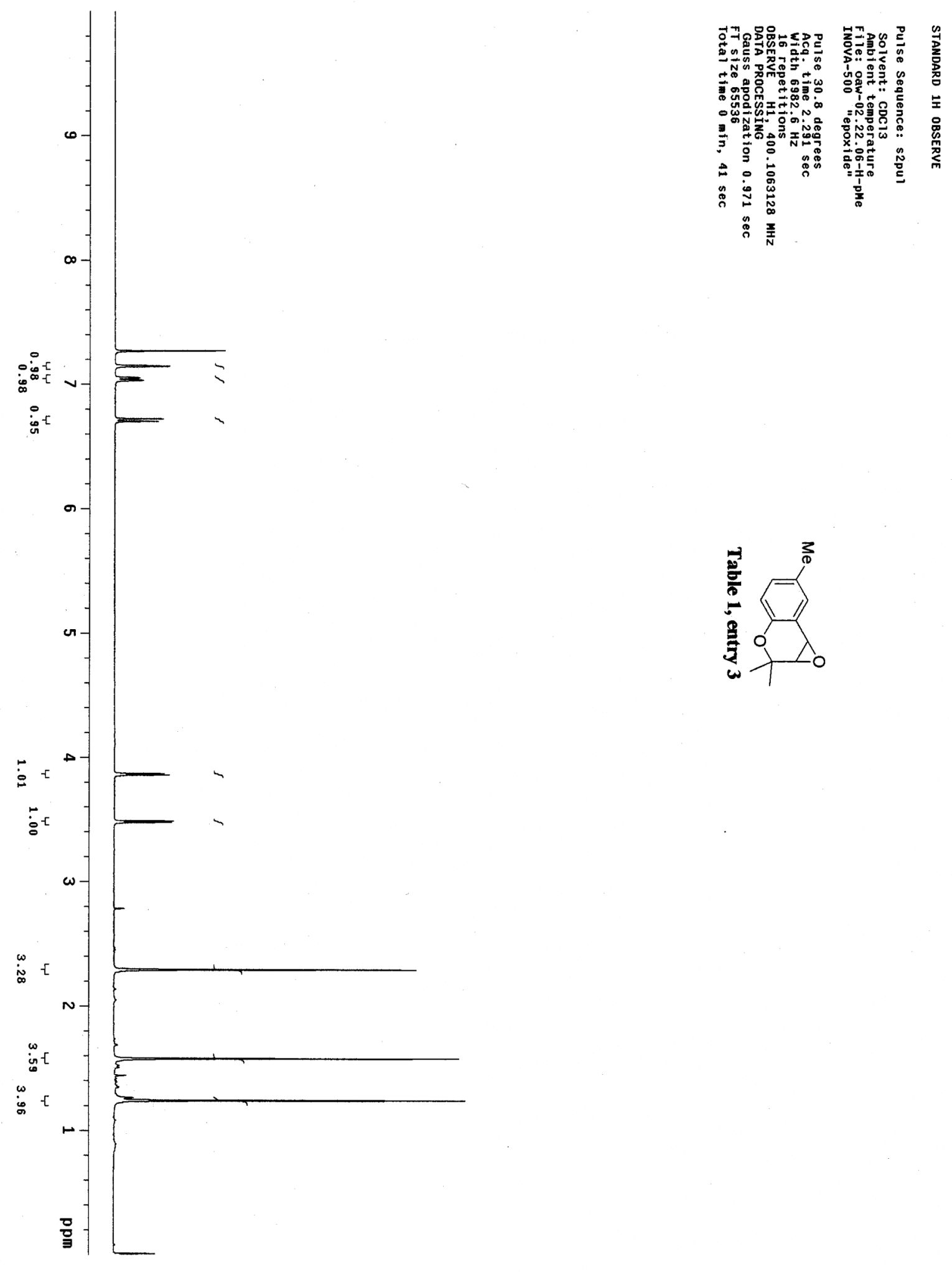

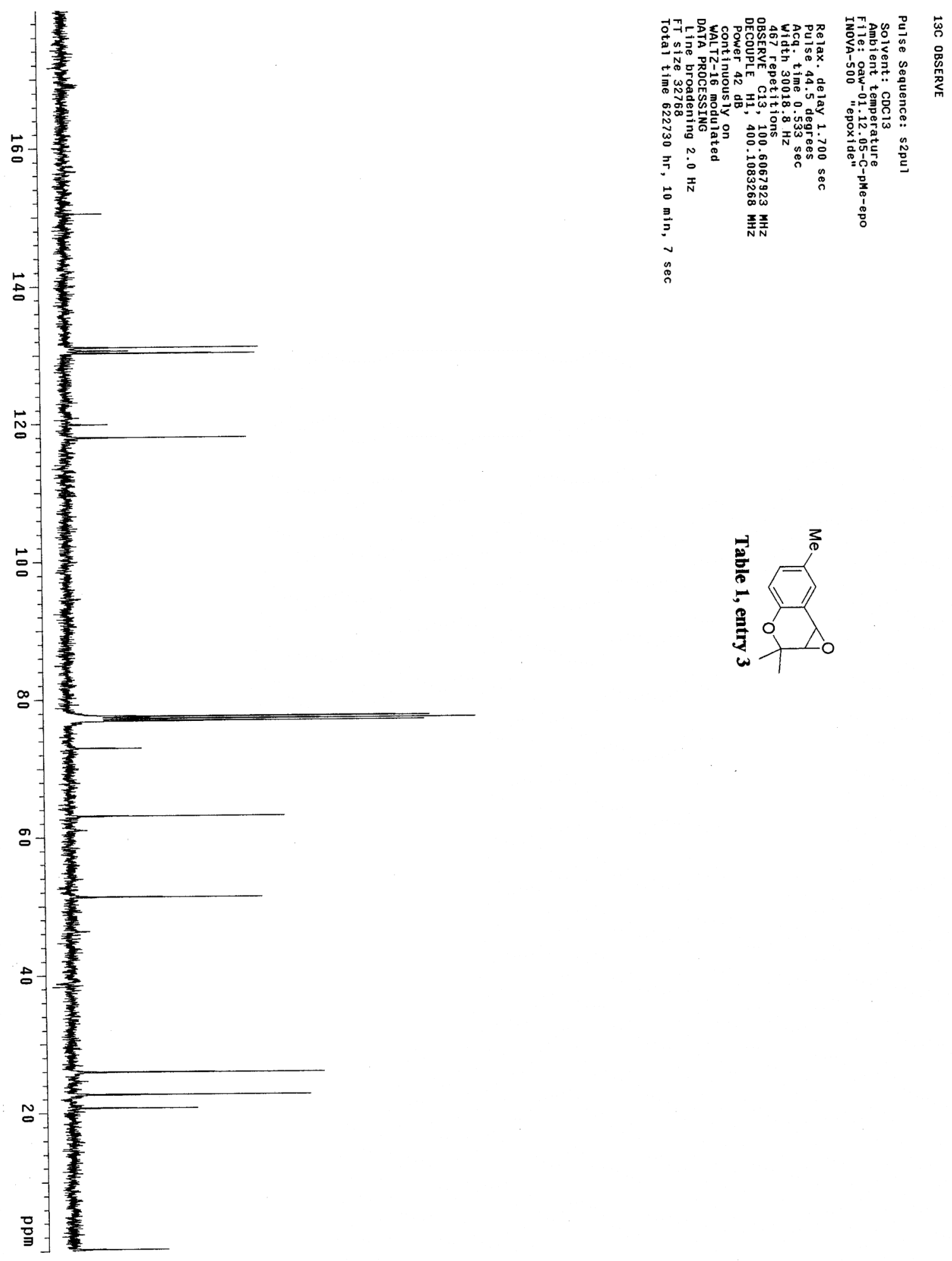


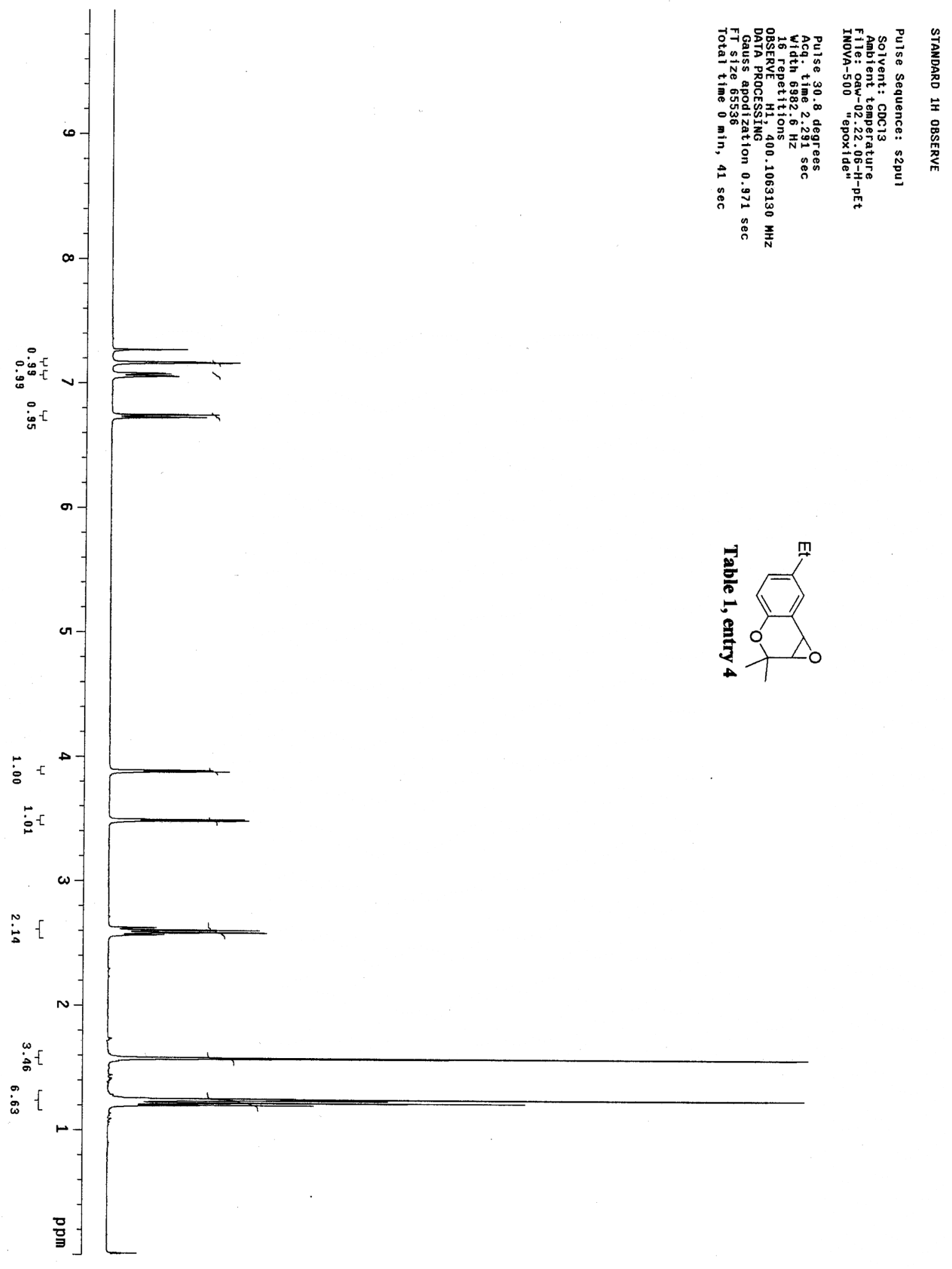



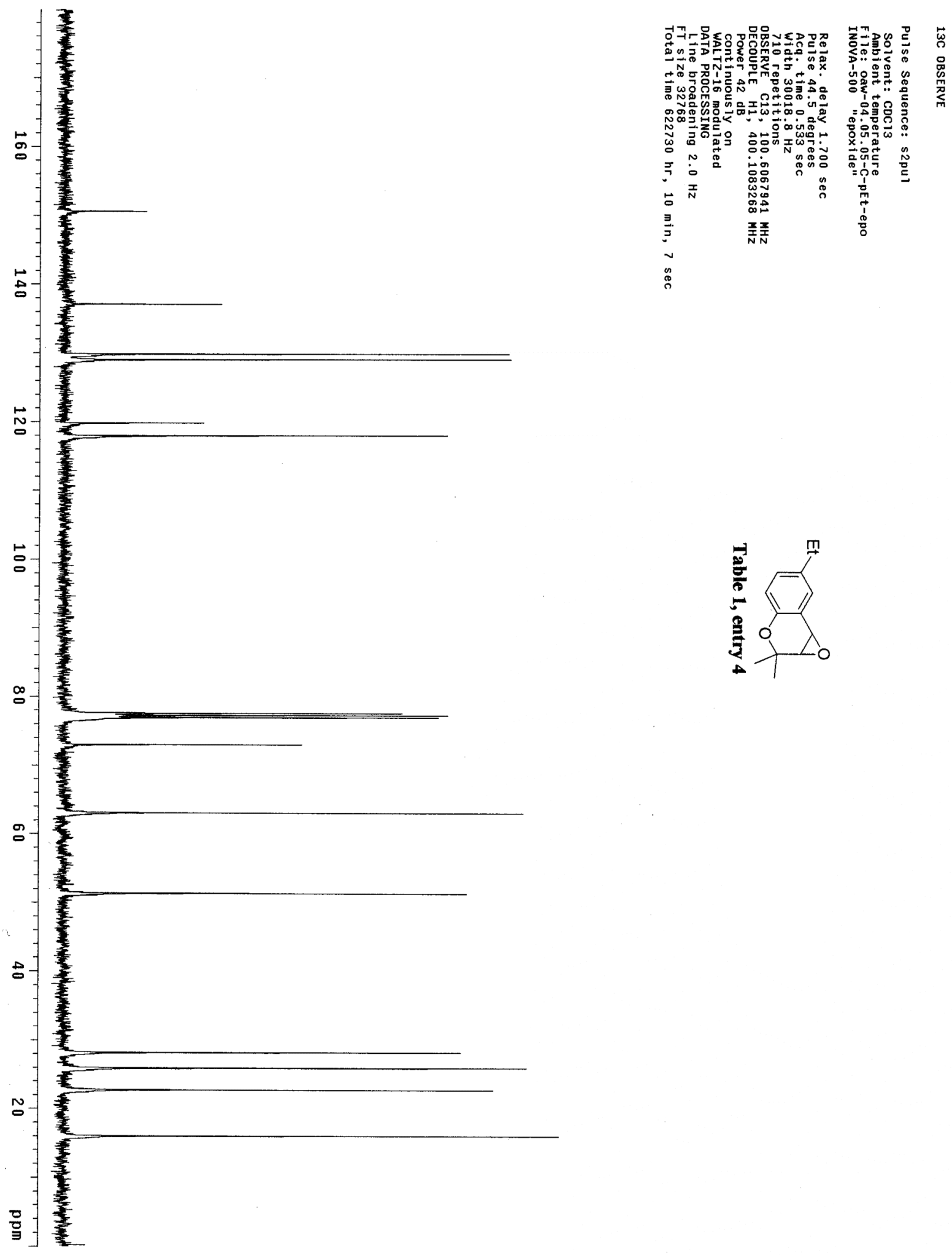


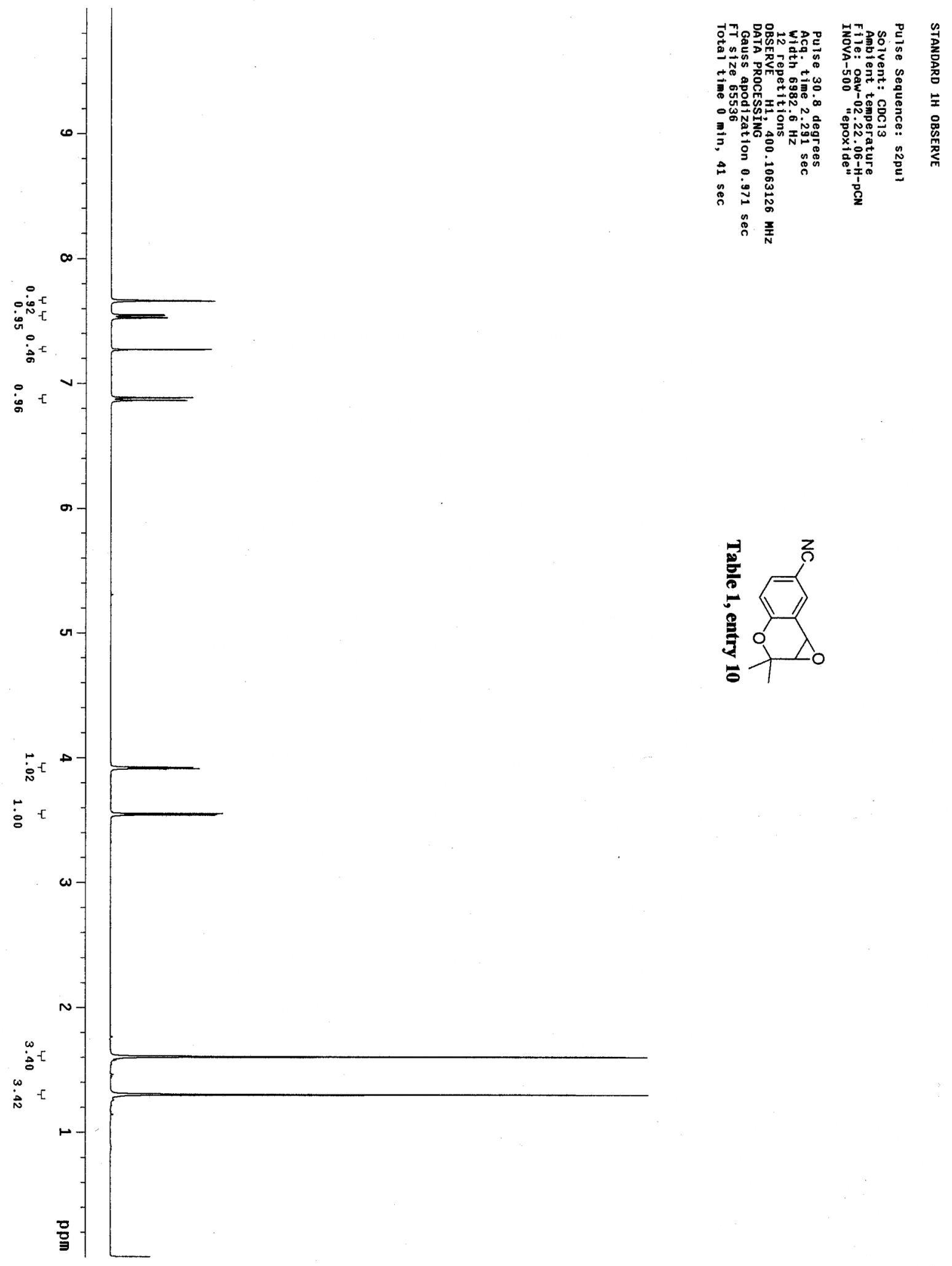




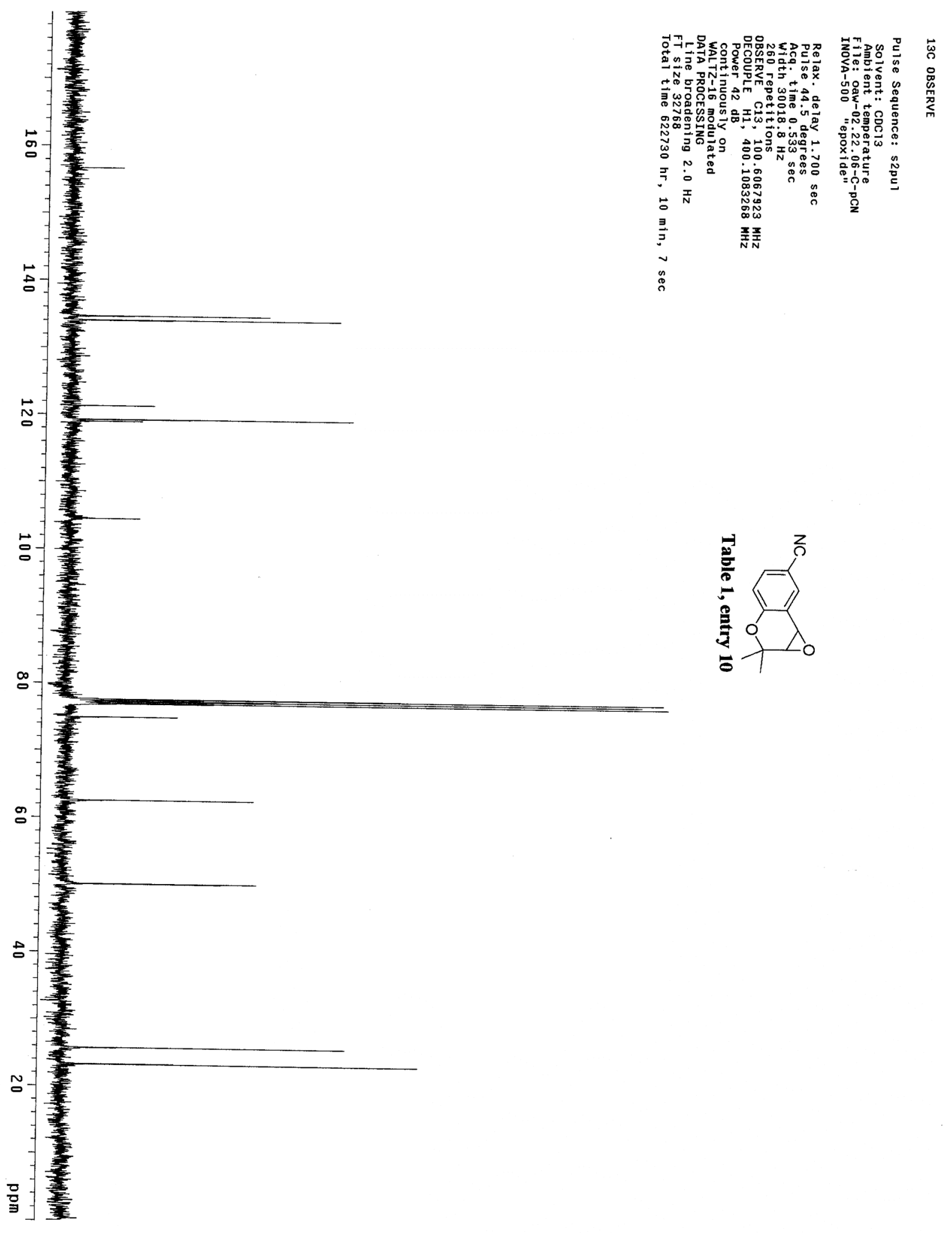




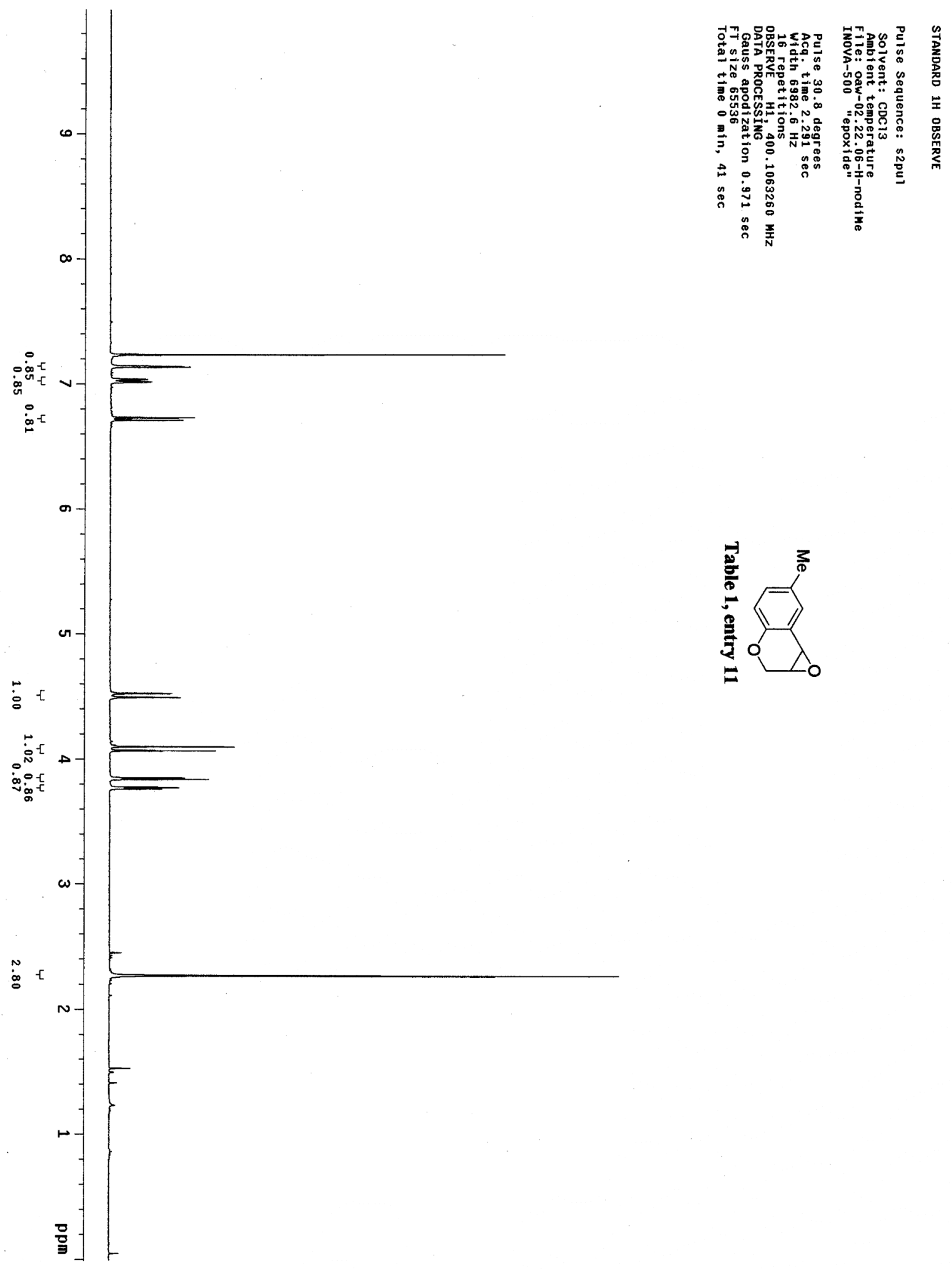



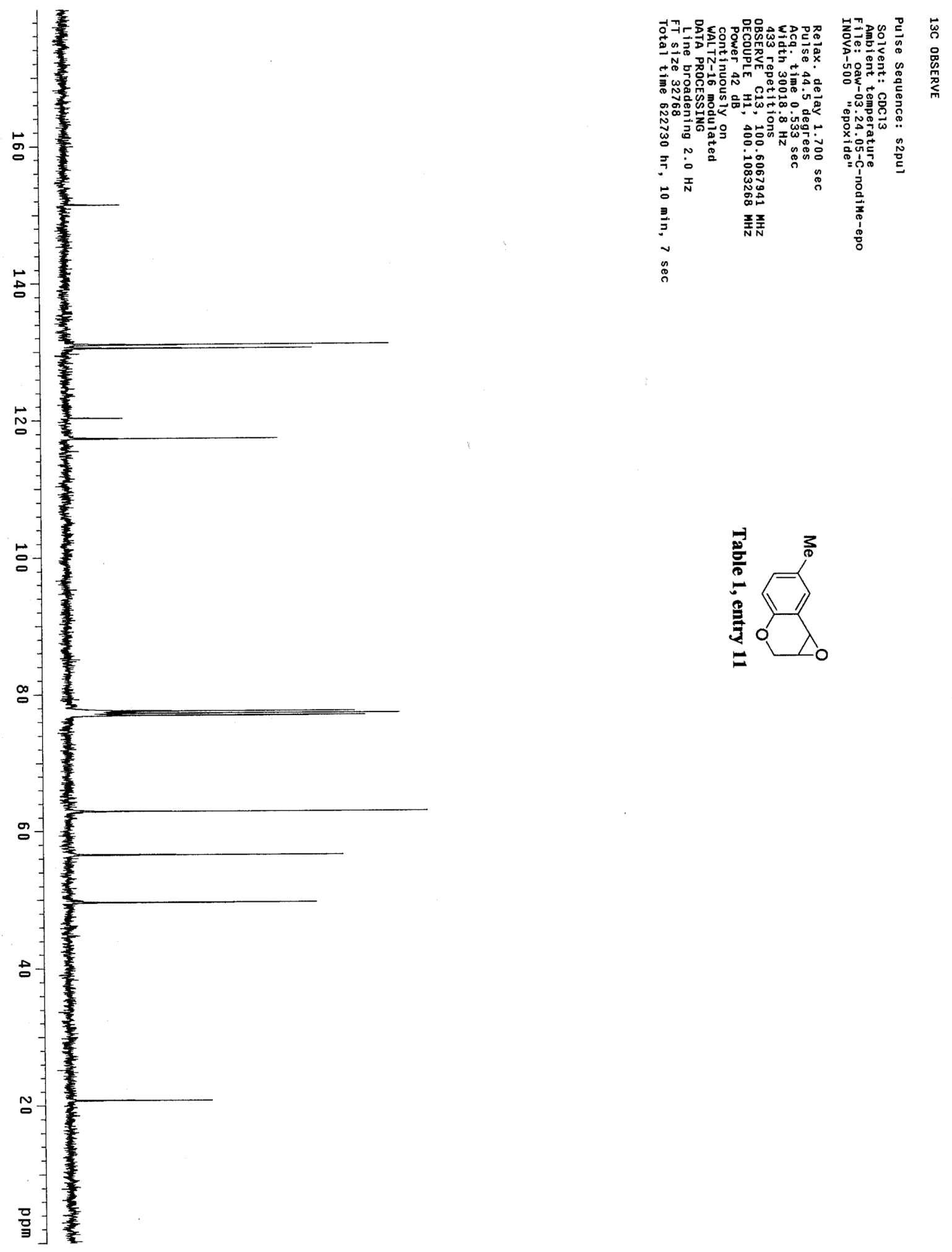


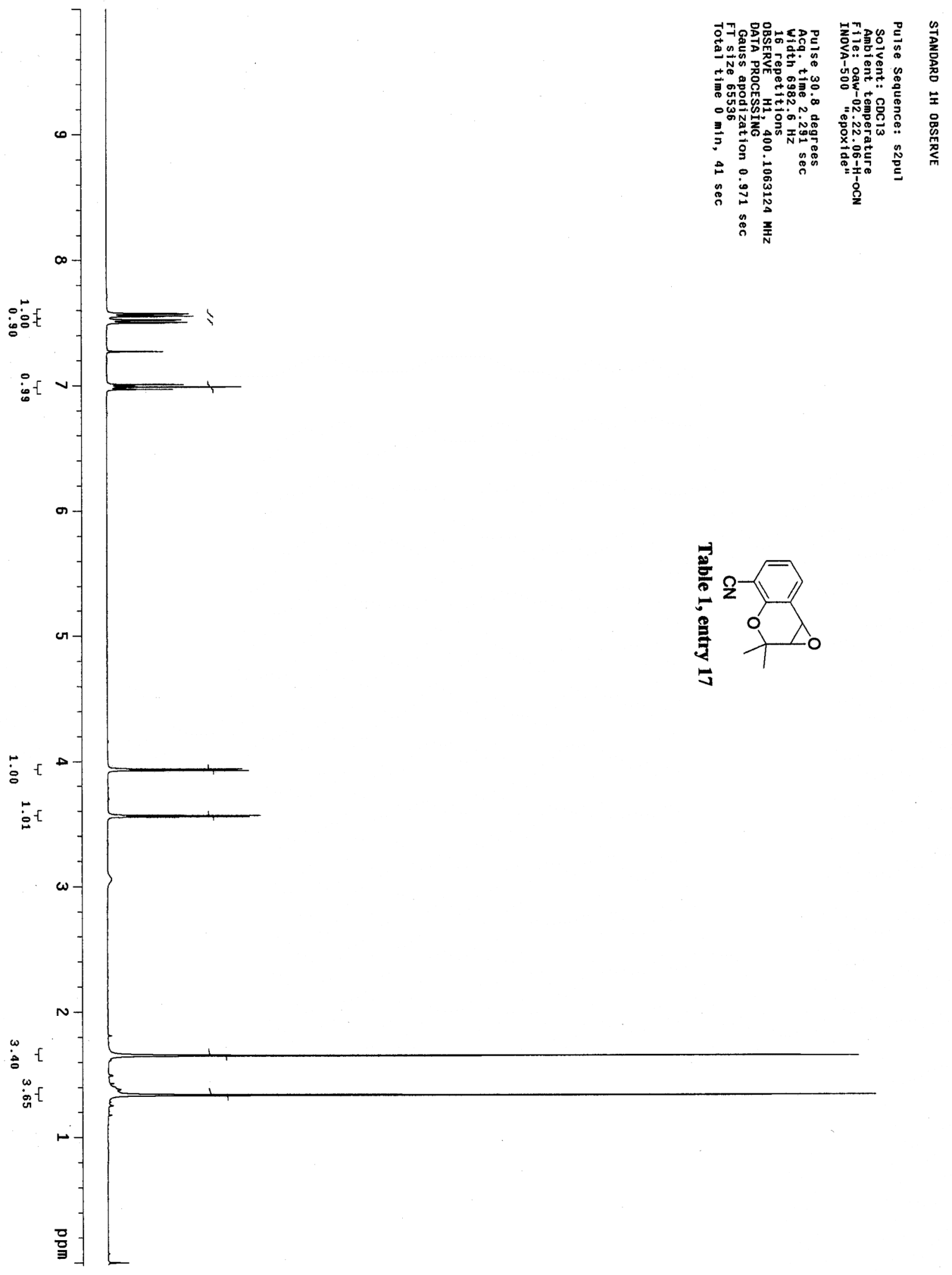



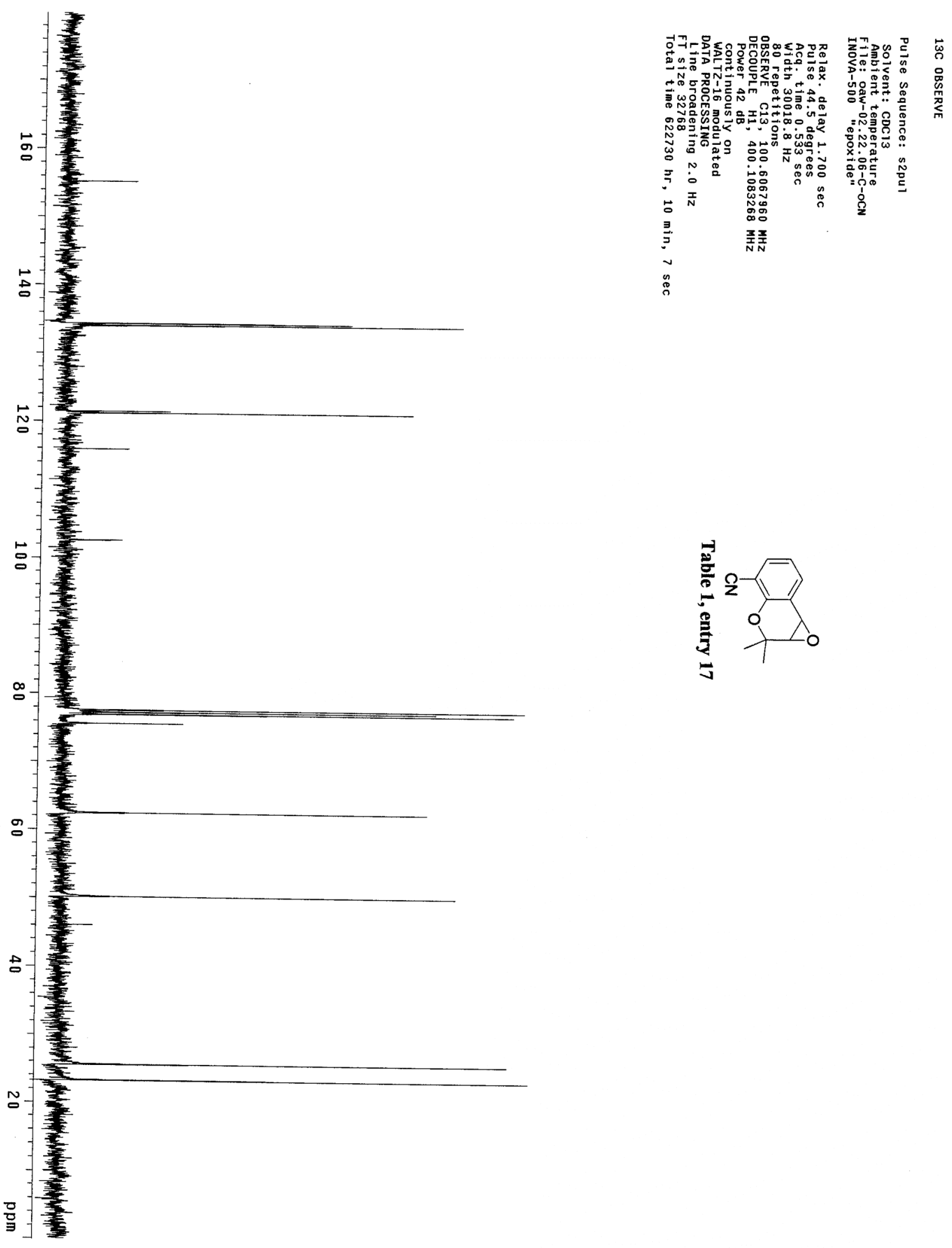